Acta Crystallographica Section E

\section{Structure Reports}

Online

ISSN 1600-5368

\section{Zaki S. Seddigi, ${ }^{a}$ G. M. Golzar Hossain $^{\text {b* }}$ and Afroza Banuc}

a Department of Chemistry, King Fahd University of Petroleum and Minerals, PO Box 5048, Dhahran 31261, Saudi Arabia, ${ }^{\mathbf{b}}$ Department of Chemistry, University of Dhaka, Dhaka 1000, Bangladesh, and ${ }^{\mathbf{c}}$ School of Chemistry, Cardiff University, Cardiff CF10 3AT, Wales

Correspondence e-mail: acsbd@yahoo.com

\section{Key indicators}

Single-crystal X-ray study

$T=150 \mathrm{~K}$

Mean $\sigma(\mathrm{C}-\mathrm{C})=0.011 \AA$

$R$ factor $=0.061$

$w R$ factor $=0.125$

Data-to-parameter ratio $=24.3$

For details of how these key indicators were automatically derived from the article, see http://journals.iucr.org/e.
(C) 2007 International Union of Crystallography All rights reserved

\title{
Tetra- $\mu_{3}$-iodo-tetrakis[(cyclohexyldiphenyl- phosphine- $\kappa$ P) copper(I)]
}

The molecule of the title compound, $\left[\mathrm{Cu}_{4} \mathrm{I}_{4}\left(\mathrm{C}_{18} \mathrm{H}_{21} \mathrm{P}\right)_{4}\right]$, which lies on a crystallographic twofold rotation axis, displays a cubane-like $\mathrm{Cu}_{4} \mathrm{I}_{4}$ core.

\section{Comment}

Phosphine complexes of copper(I) halides $\left[\left(\mathrm{Cu} X L_{n}\right)_{m}\right](X=$ halogen and $L=$ phosphine), which display a wide range of coordination geometries, are useful as catalysts, as precursors to organocopper reagents (Taylor, 1994) and as starting materials for the preparation of heterometallic complexes (Albano et al., 1995; Kudinov et al., 1993). It was proposed that the title complex, (I), has an irregular cubane structure (Churchill \& Kalra, 1974a,b,c; Churchill \& Rotella, 1977), which is confirmed in the present study. The tetranuclear molecule lies on a crystallographic twofold rotation axis (Fig. 1).

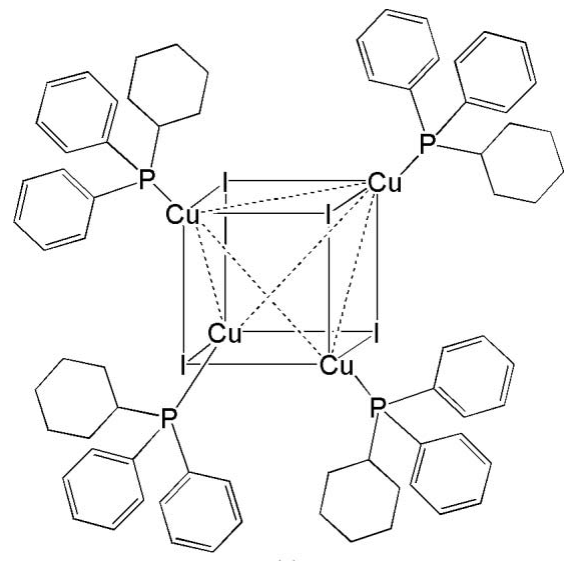

(I)

The $\mathrm{Cu}_{4} \mathrm{I}_{4}$ core is a slightly irregular cubane with alternating copper(I) and iodide ions. The $\mathrm{Cu}$ atom exists in a tetrahedral environment, being linked to three I atoms and to the $\mathrm{P}$ atom of the cyclohexyldiphenylphosphine ligand.

The copper-iodine bond lengths show a significant range of values. The average of the bond lengths is in good agreement with the values of 2.6837 (13) and 2.6767 (15) $\AA$ in the $\mathrm{Cu}_{4} \mathrm{I}_{4}$ cores of other regular cubane-like adducts (Churchill \& Kalra, 1974a,b,c; Churchill \& Rotella, 1977). The six copper-copper contact distances are similar to reported values. The iodineiodine contacts are also comparable to reported values, as are the copper-phosphorus bond distances.

There are van der Waals repulsive forces that may be responsible for the distortion of the six faces of the cubane core; the distortion manifests itself in the small $\mathrm{Cu}-\mathrm{I}-\mathrm{Cu}$ angles and in the non-planarity of the four-membered rings defining the faces of the cube.
Received 24 January 2007 Accepted 5 February 2007 


\section{Experimental}

Cyclohexyldiphenylphosphine $(0.235 \mathrm{~g}, 1 \mathrm{mmol})$ and copper(I) iodide $(0.135 \mathrm{~g}, 2 \mathrm{mmol})$ were dissolved in $30 \mathrm{ml}$ of acetone. After refluxing the mixture for one day, the hot solution was filtered. Cuboidal crystals were obtained upon recrystallization from the same solvent.

\section{Crystal data}

$\left[\mathrm{Cu}_{4} \mathrm{I}_{4}\left(\mathrm{C}_{18} \mathrm{H}_{21} \mathrm{P}\right)_{4}\right.$

$M_{r}=1835.03$

Monoclinic, $C 2 / c$

$a=22.7712$ (3) ̊

$b=15.6704(3) \AA$

$c=21.9311(5) \AA$

$\beta=116.054(1)^{\circ}$

$V=7030.5(2) \AA^{3}$

\section{Data collection}

Nonius KappaCCD diffractometer $\omega$ scans

Absorption correction: multi-scan (SORTAV; Blessing, 1995)

$T_{\min }=0.501, T_{\max }=0.578$

(expected range $=0.469-0.540)$

\section{Refinement}

Refinement on $F^{2}$

$R\left[F^{2}>2 \sigma\left(F^{2}\right)\right]=0.061$

$w R\left(F^{2}\right)=0.125$

$S=1.21$

8032 reflections

331 parameters

$$
\begin{aligned}
& Z=4 \\
& D_{x}=1.734 \mathrm{Mg} \mathrm{m}^{-3} \\
& \text { Mo } K \alpha \text { radiation } \\
& \mu=3.08 \mathrm{~mm}^{-1} \\
& T=150(2) \mathrm{K} \\
& \text { Cuboid, colorless } \\
& 0.26 \times 0.22 \times 0.20 \mathrm{~mm}
\end{aligned}
$$

63740 measured reflections 8032 independent reflections 6289 reflections with $I>2 \sigma(I)$ $R_{\text {int }}=0.127$

$\theta_{\max }=27.5^{\circ}$

$\mathrm{H}$-atom parameters constrained $w=1 /\left[\sigma^{2}\left(F_{\mathrm{o}}{ }^{2}\right)+97.862 P\right]$ where $P=\left(F_{\mathrm{o}}{ }^{2}+2 F_{\mathrm{c}}{ }^{2}\right) / 3$ $(\Delta / \sigma)_{\max }=0.001$ $\Delta \rho_{\max }=1.00{\mathrm{e} \AA^{-3}}^{-3}$ $\Delta \rho_{\min }=-0.92 \mathrm{e} \AA^{-3}$
Table 1

Selected geometric parameters $\left(\AA{ }^{\circ}\right)$.

\begin{tabular}{lrlr}
\hline $\mathrm{I} 1-\mathrm{Cu} 1$ & $2.6976(11)$ & $\mathrm{I} 2-\mathrm{Cu} 2^{\mathrm{i}}$ & $2.7667(11)$ \\
$\mathrm{I} 1-\mathrm{Cu} 1^{\mathrm{i}}$ & $2.6849(10)$ & $\mathrm{Cu} 1-\mathrm{Cu} 2$ & $2.8631(13)$ \\
$\mathrm{I} 1-\mathrm{Cu} 2$ & $2.6645(10)$ & $\mathrm{Cu} 1-\mathrm{Cu} 2^{\mathrm{i}}$ & $3.0292(13)$ \\
$\mathrm{I} 2-\mathrm{Cu} 2$ & $2.6543(11)$ & $\mathrm{Cu} 2-\mathrm{Cu} 2^{\mathrm{i}}$ & $3.0570(18)$ \\
$\mathrm{I} 2-\mathrm{Cu} 1$ & $2.7077(10)$ & & \\
$\mathrm{Cu} 1^{\mathrm{i}}-\mathrm{I} 1-\mathrm{Cu} 1$ & $70.07(4)$ & $\mathrm{I} 2-\mathrm{Cu} 2-\mathrm{I} 1$ & $115.10(4)$ \\
$\mathrm{Cu} 2-\mathrm{I} 1-\mathrm{Cu} 1^{\mathrm{i}}$ & $68.98(3)$ & $\mathrm{I} 2-\mathrm{Cu} 2-\mathrm{I} 2^{\mathrm{i}}$ & $104.49(3)$ \\
$\mathrm{Cu} 2-\mathrm{I} 1-\mathrm{Cu} 1$ & $64.54(3)$ & $\mathrm{I} 1-\mathrm{Cu} 2-\mathrm{I} 2^{\mathrm{i}}$ & $106.78(4)$ \\
$\mathrm{Cu} 2-\mathrm{I} 2-\mathrm{Cu} 1$ & $64.54(3)$ & $\mathrm{I} 2-\mathrm{Cu} 2-\mathrm{Cu} 1$ & $58.64(3)$ \\
$\mathrm{Cu} 2-\mathrm{I} 2-\mathrm{Cu} 2^{\mathrm{i}}$ & $68.62(3)$ & $\mathrm{I} 1-\mathrm{Cu} 2-\mathrm{Cu} 1$ & $58.29(3)$ \\
$\mathrm{Cu} 1-\mathrm{I} 2-\mathrm{Cu} 2^{\mathrm{i}}$ & $67.18(3)$ & $\mathrm{I} 2^{\mathrm{i}}-\mathrm{Cu} 2-\mathrm{Cu} 1$ & $107.27(4)$ \\
$\mathrm{I} 1^{\mathrm{i}}-\mathrm{Cu} 1-\mathrm{I} 1$ & $103.98(3)$ & $\mathrm{I} 2-\mathrm{Cu} 2-\mathrm{Cu} 1^{\mathrm{i}}$ & $105.64(4)$ \\
$\mathrm{I} 1^{\mathrm{i}}-\mathrm{Cu} 1-\mathrm{I} 2$ & $107.90(3)$ & $\mathrm{I} 1-\mathrm{Cu} 2-\mathrm{Cu} 1^{\mathrm{i}}$ & $55.83(3)$ \\
$\mathrm{I} 1-\mathrm{Cu} 1-\mathrm{I} 2$ & $112.27(4)$ & $\mathrm{I} 2^{\mathrm{i}}-\mathrm{Cu} 2-\mathrm{Cu} 1^{\mathrm{i}}$ & $55.48(3)$ \\
$\mathrm{I} 1^{\mathrm{i}}-\mathrm{Cu} 1-\mathrm{Cu} 2$ & $107.34(4)$ & $\mathrm{Cu} 1-\mathrm{Cu} 2-\mathrm{Cu} 1^{\mathrm{i}}$ & $63.18(4)$ \\
$\mathrm{I} 1-\mathrm{Cu} 1-\mathrm{Cu} 2$ & $57.17(3)$ & $\mathrm{I} 2-\mathrm{Cu} 2-\mathrm{Cu} 2^{\mathrm{i}}$ & $57.43(3)$ \\
$\mathrm{I} 2-\mathrm{Cu} 1-\mathrm{Cu} 2$ & $56.83(3)$ & $\mathrm{I} 1-\mathrm{Cu} 2-\mathrm{Cu} 2^{\mathrm{i}}$ & $102.56(2)$ \\
$\mathrm{I} 1^{\mathrm{i}}-\mathrm{Cu} 1-\mathrm{Cu} 2^{\mathrm{i}}$ & $55.19(3)$ & $\mathrm{I} 2^{\mathrm{i}}-\mathrm{Cu} 2-\mathrm{Cu} 2^{\mathrm{i}}$ & $53.95(3)$ \\
$\mathrm{I} 1-\mathrm{Cu} 1-\mathrm{Cu} 2^{\mathrm{i}}$ & $102.49(4)$ & $\mathrm{Cu} 1-\mathrm{Cu} 2-\mathrm{Cu} 2^{\mathrm{i}}$ & $61.45(3)$ \\
$\mathrm{I} 2-\mathrm{Cu} 1-\mathrm{Cu} 2^{\mathrm{i}}$ & $57.34(3)$ & $\mathrm{Cu} 1^{\mathrm{i}}-\mathrm{Cu} 2-\mathrm{Cu} 2^{\mathrm{i}}$ & $56.12(3)$ \\
$\mathrm{Cu} 2-\mathrm{Cu} 1-\mathrm{Cu} 2^{\mathrm{i}}$ & $62.43(4)$ & &
\end{tabular}

Symmetry code: (i) $-x+1, y,-z+\frac{3}{2}$

The data did not diffract well and the mosaicity was high so the $R_{\text {int }}$ value was high. $\mathrm{H}$ atoms were placed in calculated positions $(\mathrm{C}-\mathrm{H}=$ 0.95-1.00 $\AA$ ) and included as riding atoms, with $U_{\text {iso }}(\mathrm{H})$ values of $1.2 U_{\text {eq }}$ of the attached $\mathrm{C}$ atoms. The final difference Fourier map had a large peak/hole $1.00 \AA$ from atom $\mathrm{C} 14$..

Data collection: COLLECT (Nonius, 2000); cell refinement: SCALEPACK (Otwinowski \& Minor, 1997); data reduction: SCALEPACK and DENZO (Otwinowski \& Minor, 1997);

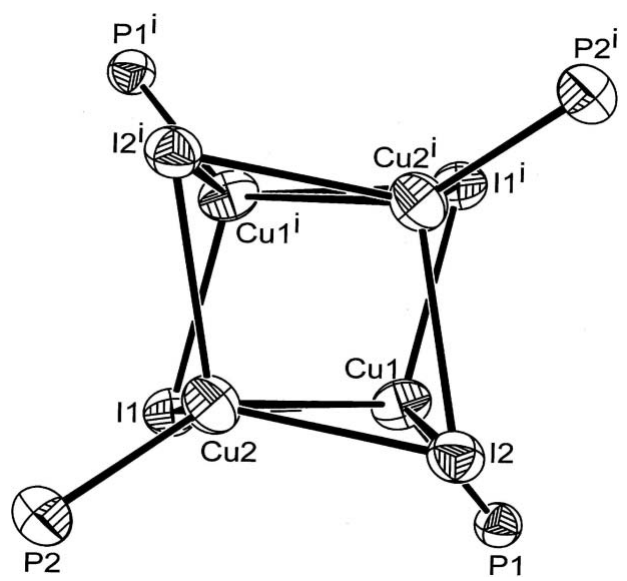

Figure 1

The central coren of (I), showing the atom-labeling scheme. Displacement ellipsoids are drawn at the $50 \%$ probability level. The symmetry code is given in Table 1 .

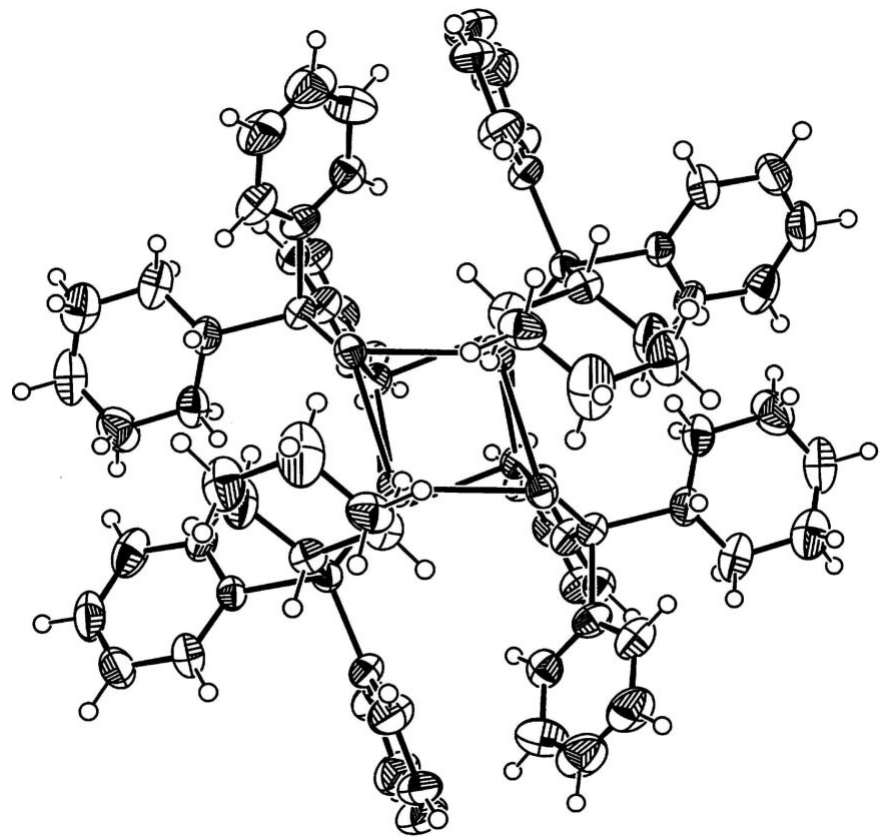

Figure 2

The molecular structure of complex (I). Displacement ellipsoids are drawn at the $50 \%$ probability level. $\mathrm{H}$ atoms are represented by circles of arbitrary size.

program(s) used to solve structure: SHELXS97 (Sheldrick, 1997); program(s) used to refine structure: SHELXL97 (Sheldrick, 1997); molecular graphics: ORTEP-3 for Windows (Farrugia, 1997); software used to prepare material for publication: Win $G X$ (Farrugia, 1999).

ZSS acknowledges King Fahd University of Petroleum and Minerals, Dhahran, Saudi Arabia, for financial support.

\section{References}

Albano, V. G., Busetto, L., Sabatino, M. C., Schmitz, P. \& Zanotti, V. (1995). J. Chem. Soc. Dalton Trans. pp. 287-2093.

Blessing, R. H. (1995). Acta Cryst. A51, 33-38.

Churchill, M. R. \& Kalra, K. L. (1974a). Inorg. Chem. 13, 1065-1071.

Churchill, M. R. \& Kalra, K. L. (1974b). Inorg. Chem. 13, 1427-1434. 


\section{metal-organic papers}

Churchill, M. R. \& Kalra, K. L. (1974c). Inorg. Chem. 13, 1899-1904.

Churchill, M. R. \& Rotella, F. J. (1977). Inorg. Chem. 16, 3267-3273.

Farrugia, L. J. (1997). J. Appl. Cryst. 30, 565.

Farrugia, L. J. (1999). J. Appl. Cryst. 32, 837-838.

Kudinov, A., Muratov, D., Rybinskaya, M. \& Turpeinen, U. (1993). Mendeleev Commun. 2, 39-40.

Nonius (2000). COLLECT. Nonius BV, Delft, The Netherlands.
Otwinowski, Z. \& Minor, W. (1997). Methods in Enzymology, Vol. 276, Macromolecular Crystallography, Part A, edited by C. W. Carter Jr \& R. M. Sweet, pp. 307-326. New York: Academic Press.

Sheldrick, G. M. (1997). SHELXS97 and SHELXL97. University of Göttingen, Germany.

Taylor, R. J. K. (1994). Organocopper Reagents. A Practical Approach. Oxford University Press. 


\title{
supporting information
}

Acta Cryst. (2007). E63, m756-m758 [https://doi.org/10.1107/S1600536807006204]

\section{Tetra- $\mu_{3}$-iodo-tetrakis[(cyclohexyldiphenylphosphine- $\left.\left.\kappa P\right) \operatorname{copper}(\mathrm{I})\right]$}

\author{
Zaki S. Seddigi, G. M. Golzar Hossain and Afroza Banu
}

Tetra- $\mu_{3}$-iodo-tetrakis [(cyclohexyldiphenylphosphine- $\left.\left.\kappa P\right) \operatorname{copper}(\mathrm{I})\right]$

Crystal data

$\left[\mathrm{Cu}_{4} \mathrm{I}_{4}\left(\mathrm{C}_{18} \mathrm{H}_{21} \mathrm{P}\right)_{4}\right]$

$M_{r}=1835.03$

Monoclinic, $C 2 / c$

Hall symbol: $-\mathrm{C} 2 \mathrm{yc}$

$a=22.7712(3) \AA$

$b=15.6704(3) \AA$

$c=21.9311(5) \AA$

$\beta=116.054(1)^{\circ}$

$V=7030.5(2) \AA^{3}$

$Z=4$

$F(000)=3616$

$D_{\mathrm{x}}=1.734 \mathrm{Mg} \mathrm{m}^{-3}$

Mo $K \alpha$ radiation, $\lambda=0.71073 \AA$

Cell parameters from 8077 reflections

$\theta=2.9-27.5^{\circ}$

$\mu=3.08 \mathrm{~mm}^{-1}$

$T=150 \mathrm{~K}$

Cuboid, colorless

$0.26 \times 0.22 \times 0.20 \mathrm{~mm}$

\section{Data collection}

Nonius KappaCCD diffractometer

Radiation source: fine-focus sealed tube

8032 independent reflections

Graphite monochromator

$\omega$ scans

Absorption correction: multi-scan

$T_{\min }=0.501, T_{\max }=0.578$

63740 measured reflections 6289 reflections with $I>2 \sigma(I)$

$R_{\text {int }}=0.127$

$\theta_{\max }=27.5^{\circ}, \theta_{\min }=3.0^{\circ}$

$h=-29 \rightarrow 29$

$k=-20 \rightarrow 19$

$l=-28 \rightarrow 28$

\section{Refinement}

Refinement on $F^{2}$

Least-squares matrix: full

$R\left[F^{2}>2 \sigma\left(F^{2}\right)\right]=0.061$

$w R\left(F^{2}\right)=0.125$

$S=1.21$

8032 reflections

331 parameters

24 restraints

Primary atom site location: structure-invariant direct methods

Secondary atom site location: difference Fourier map

Hydrogen site location: inferred from neighbouring sites

$\mathrm{H}$-atom parameters constrained

$w=1 /\left[\sigma^{2}\left(F_{\mathrm{o}}^{2}\right)+97.862 P\right]$

where $P=\left(F_{\mathrm{o}}{ }^{2}+2 F_{\mathrm{c}}{ }^{2}\right) / 3$

$(\Delta / \sigma)_{\max }=0.001$

$\Delta \rho_{\max }=1.01 \mathrm{e} \AA^{-3}$

$\Delta \rho_{\min }=-0.92$ e $\AA^{-3}$

Special details

Geometry. All e.s.d.'s (except the e.s.d. in the dihedral angle between two 1.s. planes) are estimated using the full covariance matrix. The cell e.s.d.'s are taken into account individually in the estimation of e.s.d.'s in distances, angles and torsion angles; correlations between e.s.d.'s in cell parameters are only used when they are defined by crystal symmetry. An approximate (isotropic) treatment of cell e.s.d.'s is used for estimating e.s.d.'s involving 1.s. planes. 
Refinement. Refinement of $F^{2}$ against ALL reflections. The weighted $R$-factor $w R$ and goodness of fit $S$ are based on $F^{2}$, conventional $R$-factors $R$ are based on $F$, with $F$ set to zero for negative $F^{2}$. The threshold expression of $F^{2}>\sigma\left(F^{2}\right)$ is used only for calculating $R$-factors(gt) etc. and is not relevant to the choice of reflections for refinement. $R$-factors based on $F^{2}$ are statistically about twice as large as those based on $F$, and $R$ - factors based on ALL data will be even larger.

Fractional atomic coordinates and isotropic or equivalent isotropic displacement parameters $\left(\hat{A}^{2}\right)$

\begin{tabular}{|c|c|c|c|c|}
\hline & $x$ & $y$ & $z$ & $U_{\text {iso }} * / U_{\text {eq }}$ \\
\hline I1 & $0.50557(2)$ & $0.49256(3)$ & 0.65599 (2) & $0.03452(13)$ \\
\hline $\mathrm{I} 2$ & $0.39523(2)$ & $0.69922(3)$ & $0.70308(2)$ & $0.03321(12)$ \\
\hline $\mathrm{Cu} 1$ & $0.42457(5)$ & $0.53083(6)$ & $0.71218(5)$ & $0.0394(2)$ \\
\hline $\mathrm{Cu} 2$ & $0.49587(5)$ & $0.65787(6)$ & $0.67853(5)$ & $0.0381(2)$ \\
\hline P1 & $0.33129(9)$ & $0.45435(12)$ & $0.66404(9)$ & $0.0316(4)$ \\
\hline $\mathrm{P} 2$ & $0.51053(10)$ & $0.72918(12)$ & $0.59690(10)$ & $0.0360(4)$ \\
\hline $\mathrm{C} 1$ & $0.2712(2)$ & $0.4907(3)$ & $0.58054(19)$ & $0.0334(16)$ \\
\hline $\mathrm{C} 2$ & $0.2911(2)$ & $0.5474(3)$ & $0.5445(2)$ & $0.046(2)$ \\
\hline $\mathrm{H} 2$ & 0.3349 & 0.5672 & 0.5636 & $0.055^{*}$ \\
\hline C3 & $0.2467(3)$ & $0.5752(3)$ & $0.4806(2)$ & $0.064(3)$ \\
\hline H3 & 0.2603 & 0.6140 & 0.4560 & $0.076^{*}$ \\
\hline $\mathrm{C} 4$ & $0.1825(3)$ & $0.5463(4)$ & $0.4526(2)$ & $0.064(3)$ \\
\hline H4 & 0.1522 & 0.5653 & 0.4089 & $0.076^{*}$ \\
\hline $\mathrm{C} 5$ & $0.1626(2)$ & $0.4896(4)$ & $0.4886(3)$ & $0.056(2)$ \\
\hline H5 & 0.1187 & 0.4698 & 0.4695 & $0.068 *$ \\
\hline C6 & $0.2069(2)$ & $0.4618(3)$ & $0.5526(2)$ & $0.050(2)$ \\
\hline H6 & 0.1934 & 0.4230 & 0.5772 & $0.060 *$ \\
\hline $\mathrm{C} 7$ & $0.2815(3)$ & $0.4538(3)$ & $0.7114(3)$ & 0.0413 (18) \\
\hline $\mathrm{C} 8$ & 0.2564 & $0.5324(3)$ & 0.7178 & $0.053(2)$ \\
\hline H8 & 0.2666 & 0.5822 & 0.6997 & $0.063^{*}$ \\
\hline $\mathrm{C} 9$ & 0.2163 & $0.5379(4)$ & $0.7505(3)$ & $0.071(3)$ \\
\hline H9 & 0.1991 & 0.5916 & 0.7549 & $0.085^{*}$ \\
\hline C10 & 0.2013 & $0.4650(5)$ & $0.7769(3)$ & $0.078(3)$ \\
\hline H10 & 0.1740 & 0.4688 & 0.7993 & $0.094^{*}$ \\
\hline C11 & $0.2265(3)$ & $0.3864(4)$ & $0.7706(3)$ & $0.061(3)$ \\
\hline H11 & 0.2162 & 0.3365 & 0.7886 & $0.073^{*}$ \\
\hline $\mathrm{C} 12$ & $0.2665(3)$ & $0.3808(3)$ & $0.7378(3)$ & $0.059(2)$ \\
\hline H12 & 0.2837 & 0.3271 & 0.7335 & $0.071^{*}$ \\
\hline $\mathrm{C} 13$ & $0.3412(3)$ & $0.3405(4)$ & $0.6489(3)$ & $0.0362(16)$ \\
\hline H13 & 0.3001 & 0.3109 & 0.6426 & $0.043^{*}$ \\
\hline $\mathrm{C} 14$ & $0.3493(5)$ & $0.3264(4)$ & 0.5848 & $0.056(2)$ \\
\hline H14A & 0.3879 & 0.3585 & 0.5878 & $0.067^{*}$ \\
\hline H14B & 0.3103 & 0.3487 & 0.5455 & $0.067 *$ \\
\hline C15 & $0.3578(5)$ & $0.2322(5)$ & $0.5734(3)$ & $0.063(3)$ \\
\hline $\mathrm{H} 15 \mathrm{~A}$ & 0.3662 & 0.2259 & 0.5330 & $0.075^{*}$ \\
\hline H15B & 0.3170 & 0.2012 & 0.5645 & $0.075^{*}$ \\
\hline C16 & $0.4143(5)$ & $0.1931(6)$ & $0.6349(4)$ & $0.077(3)$ \\
\hline H16A & 0.4171 & 0.1313 & 0.6272 & $0.093^{*}$ \\
\hline H16B & 0.4559 & 0.2200 & 0.6412 & $0.093^{*}$ \\
\hline C17 & $0.4044(4)$ & $0.2062(4)$ & $0.6986(3)$ & $0.051(2)$ \\
\hline
\end{tabular}




\begin{tabular}{|c|c|c|c|c|}
\hline H17A & 0.3648 & 0.1748 & 0.6939 & $0.061 *$ \\
\hline H17B & 0.4423 & 0.1826 & 0.7383 & $0.061 *$ \\
\hline $\mathrm{C} 18$ & 0.3967 (4) & 0.3002 (4) & 0.7104 (3) & 0.0455 (19) \\
\hline $\mathrm{H} 18 \mathrm{~A}$ & 0.4381 & 0.3304 & 0.7200 & $0.055^{*}$ \\
\hline H18B & 0.3879 & 0.3065 & 0.7505 & $0.055^{*}$ \\
\hline C19 & $0.5802(2)$ & $0.6838(3)$ & 0.5874 (3) & $0.045(2)$ \\
\hline $\mathrm{C} 20$ & 0.5708 (3) & $0.6110(3)$ & 0.5480 & $0.055(2)$ \\
\hline $\mathrm{H} 20$ & 0.5281 & 0.5886 & 0.5230 & $0.066^{*}$ \\
\hline $\mathrm{C} 21$ & $0.6240(4)$ & $0.5710(3)$ & 0.5451 (3) & $0.070(3)$ \\
\hline $\mathrm{H} 21$ & 0.6176 & 0.5212 & 0.5181 & $0.084 *$ \\
\hline $\mathrm{C} 22$ & $0.6866(3)$ & 0.6037 (4) & $0.5816(4)$ & $0.081(4)$ \\
\hline $\mathrm{H} 22$ & 0.7229 & 0.5764 & 0.5797 & $0.097 *$ \\
\hline $\mathrm{C} 23$ & 0.6959 (2) & 0.6765 (4) & $0.6210(3)$ & $0.072(3)$ \\
\hline $\mathrm{H} 23$ & 0.7387 & 0.6989 & 0.6460 & $0.086^{*}$ \\
\hline $\mathrm{C} 24$ & 0.6427 (3) & $0.7166(3)$ & 0.6239 (3) & $0.048(2)$ \\
\hline $\mathrm{H} 24$ & 0.6491 & 0.7663 & 0.6509 & $0.057 *$ \\
\hline $\mathrm{C} 25$ & $0.5256(3)$ & $0.8451(2)$ & $0.6111(3)$ & $0.047(2)$ \\
\hline $\mathrm{C} 26$ & 0.5547 (3) & 0.8937 (3) & 0.5788 (3) & $0.0433(19)$ \\
\hline $\mathrm{H} 26$ & 0.5708 & 0.8668 & 0.5504 & $0.052^{*}$ \\
\hline $\mathrm{C} 27$ & 0.5602 (3) & $0.9816(3)$ & 0.5881 (3) & $0.054(2)$ \\
\hline $\mathrm{H} 27$ & 0.5801 & 1.0147 & 0.5661 & $0.065^{*}$ \\
\hline $\mathrm{C} 28$ & $0.5366(3)$ & 1.0209 (2) & 0.6297 (3) & $0.048(2)$ \\
\hline $\mathrm{H} 28$ & 0.5404 & 1.0810 & 0.6361 & $0.057^{*}$ \\
\hline $\mathrm{C} 29$ & 0.5075 (3) & $0.9724(3)$ & $0.6620(3)$ & $0.052(2)$ \\
\hline H29 & 0.4913 & 0.9993 & 0.6904 & $0.062^{*}$ \\
\hline $\mathrm{C} 30$ & 0.5019 (3) & $0.8845(3)$ & $0.6526(3)$ & $0.0422(19)$ \\
\hline $\mathrm{H} 30$ & 0.4820 & 0.8513 & 0.6747 & $0.051^{*}$ \\
\hline $\mathrm{C} 31$ & $0.4444(3)$ & $0.7277(6)$ & $0.5117(3)$ & $0.051(2)$ \\
\hline H31 & 0.4358 & 0.6652 & 0.5034 & $0.062 *$ \\
\hline C32 & 0.4591 (4) & $0.7531(6)$ & $0.4546(3)$ & $0.060(3)$ \\
\hline $\mathrm{H} 32 \mathrm{~A}$ & 0.4936 & 0.7148 & 0.4543 & $0.072 *$ \\
\hline H32B & 0.4770 & 0.8117 & 0.4631 & $0.072 *$ \\
\hline C33 & $0.4022(4)$ & 0.7507 (6) & $0.3855(3)$ & 0.068 (3) \\
\hline H33A & 0.4137 & 0.7838 & 0.3539 & $0.081 *$ \\
\hline H33B & 0.3949 & 0.6909 & 0.3695 & $0.081 *$ \\
\hline C34 & 0.3397 (4) & $0.7850(6)$ & $0.3821(4)$ & $0.074(3)$ \\
\hline $\mathrm{H} 34 \mathrm{~A}$ & 0.3035 & 0.7676 & 0.3384 & $0.088^{*}$ \\
\hline H34B & 0.3418 & 0.8481 & 0.3826 & $0.088^{*}$ \\
\hline C35 & $0.3240(3)$ & $0.7565(7)$ & 0.4384 (4) & $0.075(3)$ \\
\hline H35A & 0.2887 & 0.7930 & 0.4387 & $0.090^{*}$ \\
\hline $\mathrm{H} 35 \mathrm{C}$ & 0.3072 & 0.6973 & 0.4291 & $0.090^{*}$ \\
\hline C36 & 0.3807 (3) & $0.7593(6)$ & $0.5073(3)$ & $0.052(2)$ \\
\hline $\mathrm{H} 36 \mathrm{C}$ & 0.3867 & 0.8191 & 0.5236 & $0.062 *$ \\
\hline H36A & 0.3696 & 0.7250 & 0.5387 & $0.062 *$ \\
\hline
\end{tabular}


Atomic displacement parameters $\left(\AA^{2}\right)$

\begin{tabular}{|c|c|c|c|c|c|c|}
\hline & $U^{11}$ & $U^{22}$ & $U^{33}$ & $U^{12}$ & $U^{13}$ & $U^{23}$ \\
\hline I1 & $0.0349(3)$ & $0.0310(2)$ & $0.0335(2)$ & $-0.0004(2)$ & $0.0113(2)$ & $-0.00226(19)$ \\
\hline $\mathrm{I} 2$ & $0.0356(3)$ & $0.0336(2)$ & $0.0289(2)$ & $0.0052(2)$ & 0.01262 (19) & 0.00124 (19) \\
\hline $\mathrm{Cu} 1$ & $0.0346(5)$ & $0.0368(5)$ & $0.0397(5)$ & $-0.0026(4)$ & 0.0099 (4) & $-0.0012(4)$ \\
\hline $\mathrm{Cu} 2$ & $0.0452(6)$ & $0.0348(5)$ & $0.0365(5)$ & $-0.0001(4)$ & $0.0199(4)$ & $0.0016(4)$ \\
\hline $\mathrm{P} 1$ & $0.0307(10)$ & $0.0329(9)$ & $0.0297(9)$ & $0.0001(8)$ & $0.0118(8)$ & $-0.0014(8)$ \\
\hline $\mathrm{P} 2$ & $0.0447(12)$ & $0.0320(10)$ & $0.0338(10)$ & $-0.0043(9)$ & $0.0196(9)$ & $-0.0018(8)$ \\
\hline $\mathrm{C} 1$ & $0.033(4)$ & $0.035(4)$ & $0.028(3)$ & $0.002(3)$ & $0.010(3)$ & $-0.002(3)$ \\
\hline $\mathrm{C} 2$ & $0.051(5)$ & $0.054(5)$ & $0.030(4)$ & $0.000(4)$ & $0.016(4)$ & $0.004(4)$ \\
\hline $\mathrm{C} 3$ & $0.087(8)$ & $0.063(6)$ & $0.039(5)$ & $0.011(6)$ & $0.026(5)$ & $0.015(4)$ \\
\hline $\mathrm{C} 4$ & $0.065(7)$ & $0.076(7)$ & $0.032(4)$ & $0.027(5)$ & $0.004(4)$ & -0.005 (4) \\
\hline $\mathrm{C} 5$ & $0.038(5)$ & $0.071(6)$ & $0.049(5)$ & $0.003(5)$ & 0.009 (4) & $-0.014(5)$ \\
\hline C6 & $0.047(5)$ & $0.056(5)$ & $0.034(4)$ & $-0.001(4)$ & $0.006(4)$ & $-0.001(4)$ \\
\hline $\mathrm{C} 7$ & $0.043(5)$ & $0.052(5)$ & $0.034(4)$ & $-0.003(4)$ & $0.021(4)$ & $-0.004(4)$ \\
\hline $\mathrm{C} 8$ & $0.063(6)$ & $0.054(5)$ & $0.050(5)$ & $0.003(5)$ & $0.033(5)$ & $-0.004(4)$ \\
\hline $\mathrm{C} 9$ & $0.082(8)$ & $0.078(7)$ & $0.066(7)$ & $0.010(6)$ & $0.044(6)$ & $-0.003(6)$ \\
\hline $\mathrm{C} 10$ & $0.071(8)$ & $0.109(9)$ & $0.080(8)$ & $0.009(7)$ & $0.055(7)$ & $0.000(7)$ \\
\hline $\mathrm{C} 11$ & $0.045(5)$ & $0.089(7)$ & $0.057(6)$ & $0.006(5)$ & $0.030(5)$ & $0.014(5)$ \\
\hline $\mathrm{C} 12$ & $0.066(7)$ & $0.064(6)$ & $0.059(6)$ & $0.005(5)$ & $0.038(5)$ & $0.005(5)$ \\
\hline $\mathrm{C} 13$ & $0.040(4)$ & $0.031(4)$ & $0.038(4)$ & $-0.002(3)$ & $0.018(3)$ & $-0.001(3)$ \\
\hline C14 & $0.069(6)$ & $0.052(5)$ & $0.036(4)$ & $0.009(5)$ & $0.013(4)$ & $-0.006(4)$ \\
\hline $\mathrm{C} 15$ & $0.076(7)$ & $0.048(5)$ & $0.051(5)$ & $0.011(5)$ & $0.016(5)$ & $-0.018(4)$ \\
\hline $\mathrm{C} 16$ & $0.094(8)$ & $0.058(6)$ & $0.062(6)$ & $0.032(6)$ & $0.017(6)$ & $-0.004(5)$ \\
\hline $\mathrm{C} 17$ & $0.043(5)$ & $0.045(5)$ & $0.063(6)$ & $0.015(4)$ & $0.022(4)$ & $0.015(4)$ \\
\hline C18 & $0.051(5)$ & $0.038(4)$ & $0.046(5)$ & $-0.001(4)$ & $0.021(4)$ & $0.001(4)$ \\
\hline C19 & $0.061(6)$ & $0.035(4)$ & $0.051(5)$ & $-0.002(4)$ & $0.036(4)$ & $-0.002(4)$ \\
\hline $\mathrm{C} 20$ & $0.078(7)$ & $0.049(5)$ & $0.051(5)$ & $-0.002(5)$ & $0.039(5)$ & $-0.005(4)$ \\
\hline $\mathrm{C} 21$ & $0.091(9)$ & $0.058(6)$ & $0.072(7)$ & $0.021(6)$ & $0.046(7)$ & $-0.005(5)$ \\
\hline $\mathrm{C} 22$ & $0.098(10)$ & $0.083(8)$ & $0.080(8)$ & $0.039(7)$ & $0.056(8)$ & $0.019(7)$ \\
\hline $\mathrm{C} 23$ & $0.060(7)$ & $0.060(6)$ & $0.103(9)$ & $0.001(5)$ & $0.041(6)$ & $0.004(6)$ \\
\hline $\mathrm{C} 24$ & $0.053(5)$ & $0.041(4)$ & $0.045(5)$ & $0.006(4)$ & 0.019 (4) & $0.009(4)$ \\
\hline $\mathrm{C} 25$ & $0.062(6)$ & $0.036(4)$ & $0.048(5)$ & $-0.006(4)$ & $0.030(4)$ & $-0.003(4)$ \\
\hline $\mathrm{C} 26$ & $0.050(5)$ & $0.039(4)$ & $0.044(5)$ & $-0.008(4)$ & $0.023(4)$ & $-0.006(4)$ \\
\hline $\mathrm{C} 27$ & $0.075(7)$ & $0.038(4)$ & $0.060(5)$ & $-0.002(4)$ & $0.040(5)$ & 0.008 (4) \\
\hline $\mathrm{C} 28$ & $0.048(5)$ & $0.034(4)$ & $0.056(5)$ & $-0.012(4)$ & $0.017(4)$ & $-0.007(4)$ \\
\hline $\mathrm{C} 29$ & $0.074(6)$ & $0.035(4)$ & $0.060(5)$ & $0.006(4)$ & $0.042(5)$ & $-0.002(4)$ \\
\hline $\mathrm{C} 30$ & $0.059(5)$ & $0.035(4)$ & $0.045(5)$ & $-0.006(4)$ & $0.034(4)$ & 0.000 \\
\hline C31 & $0.053(5)$ & $0.064(6)$ & $0.033(4)$ & $-0.013(5)$ & $0.016(4)$ & $0.001(4)$ \\
\hline $\mathrm{C} 32$ & $0.088(8)$ & $0.049(5)$ & $0.039(5)$ & $-0.001(5)$ & $0.024(5)$ & $-0.001(4)$ \\
\hline $\mathrm{C} 33$ & $0.089(8)$ & $0.055(6)$ & $0.054(6)$ & $-0.020(6)$ & $0.027(6)$ & $0.004(5)$ \\
\hline C34 & $0.100(9)$ & $0.050(6)$ & $0.042(5)$ & $-0.003(6)$ & $0.005(5)$ & $-0.004(4)$ \\
\hline $\mathrm{C} 35$ & $0.057(6)$ & $0.084(8)$ & $0.067(7)$ & $0.003(6)$ & $0.010(5)$ & $0.024(6)$ \\
\hline $\mathrm{C} 36$ & $0.059(6)$ & $0.047(5)$ & $0.042(5)$ & -0.008 (4) & $0.015(4)$ & $-0.007(4)$ \\
\hline
\end{tabular}


Geometric parameters $\left(\AA,{ }^{\circ}\right)$

\begin{tabular}{|c|c|c|c|}
\hline $\mathrm{I} 1-\mathrm{Cu} 1$ & $2.6976(11)$ & $\mathrm{C} 15-\mathrm{H} 15 \mathrm{~A}$ & 0.9900 \\
\hline $\mathrm{I} 1-\mathrm{Cu} 1^{\mathrm{i}}$ & $2.6849(10)$ & C15-H15B & 0.9900 \\
\hline $\mathrm{I} 1-\mathrm{Cu} 2$ & $2.6645(10)$ & $\mathrm{C} 16-\mathrm{C} 17$ & $1.523(8)$ \\
\hline $\mathrm{I} 2-\mathrm{Cu} 2$ & $2.6543(11)$ & $\mathrm{C} 16-\mathrm{H} 16 \mathrm{~A}$ & 0.9900 \\
\hline $\mathrm{I} 2-\mathrm{Cu} 1$ & $2.7077(10)$ & C16-H16B & 0.9900 \\
\hline $\mathrm{I} 2-\mathrm{Cu} 2^{\mathrm{i}}$ & $2.7667(11)$ & $\mathrm{C} 17-\mathrm{C} 18$ & $1.519(7)$ \\
\hline $\mathrm{Cu} 1-\mathrm{P} 1$ & $2.257(2)$ & $\mathrm{C} 17-\mathrm{H} 17 \mathrm{~A}$ & 0.9900 \\
\hline $\mathrm{Cu} 1-\mathrm{I}^{\mathrm{i}}$ & $2.6849(10)$ & С17-H17B & 0.9900 \\
\hline $\mathrm{Cu} 1-\mathrm{Cu} 2$ & $2.8631(13)$ & $\mathrm{C} 18-\mathrm{H} 18 \mathrm{~A}$ & 0.9900 \\
\hline $\mathrm{Cu} 1-\mathrm{Cu} 2^{\mathrm{i}}$ & $3.0292(13)$ & C18-H18B & 0.9900 \\
\hline $\mathrm{Cu} 2-\mathrm{P} 2$ & $2.256(2)$ & $\mathrm{C} 19-\mathrm{C} 20$ & 1.3900 \\
\hline $\mathrm{Cu} 2-\mathrm{I} 2^{\mathrm{i}}$ & $2.7667(11)$ & $\mathrm{C} 19-\mathrm{C} 24$ & 1.3900 \\
\hline $\mathrm{Cu} 2-\mathrm{Cu} 1^{\mathrm{i}}$ & $3.0292(13)$ & $\mathrm{C} 20-\mathrm{C} 21$ & 1.3900 \\
\hline $\mathrm{Cu} 2-\mathrm{Cu} 2^{\mathrm{i}}$ & $3.0570(18)$ & $\mathrm{C} 20-\mathrm{H} 20$ & 0.9500 \\
\hline $\mathrm{P} 1-\mathrm{C} 1$ & $1.830(4)$ & $\mathrm{C} 21-\mathrm{C} 22$ & 1.3900 \\
\hline $\mathrm{P} 1-\mathrm{C} 7$ & $1.844(4)$ & $\mathrm{C} 21-\mathrm{H} 21$ & 0.9500 \\
\hline $\mathrm{P} 1-\mathrm{C} 13$ & $1.846(6)$ & $\mathrm{C} 22-\mathrm{C} 23$ & 1.3900 \\
\hline $\mathrm{P} 2-\mathrm{C} 31$ & $1.814(7)$ & $\mathrm{C} 22-\mathrm{H} 22$ & 0.9500 \\
\hline $\mathrm{P} 2-\mathrm{C} 19$ & $1.831(5)$ & $\mathrm{C} 23-\mathrm{C} 24$ & 1.3900 \\
\hline $\mathrm{P} 2-\mathrm{C} 25$ & $1.849(4)$ & $\mathrm{C} 23-\mathrm{H} 23$ & 0.9500 \\
\hline $\mathrm{C} 1-\mathrm{C} 2$ & 1.3900 & $\mathrm{C} 24-\mathrm{H} 24$ & 0.9500 \\
\hline $\mathrm{C} 1-\mathrm{C} 6$ & 1.3900 & $\mathrm{C} 25-\mathrm{C} 26$ & 1.3900 \\
\hline $\mathrm{C} 2-\mathrm{C} 3$ & 1.3900 & $\mathrm{C} 25-\mathrm{C} 30$ & 1.3900 \\
\hline $\mathrm{C} 2-\mathrm{H} 2$ & 0.9500 & $\mathrm{C} 26-\mathrm{C} 27$ & 1.3900 \\
\hline $\mathrm{C} 3-\mathrm{C} 4$ & 1.3900 & $\mathrm{C} 26-\mathrm{H} 26$ & 0.9500 \\
\hline $\mathrm{C} 3-\mathrm{H} 3$ & 0.9500 & $\mathrm{C} 27-\mathrm{C} 28$ & 1.3900 \\
\hline $\mathrm{C} 4-\mathrm{C} 5$ & 1.3900 & $\mathrm{C} 27-\mathrm{H} 27$ & 0.9500 \\
\hline $\mathrm{C} 4-\mathrm{H} 4$ & 0.9500 & $\mathrm{C} 28-\mathrm{C} 29$ & 1.3900 \\
\hline $\mathrm{C} 5-\mathrm{C} 6$ & 1.3900 & $\mathrm{C} 28-\mathrm{H} 28$ & 0.9500 \\
\hline $\mathrm{C} 5-\mathrm{H} 5$ & 0.9500 & $\mathrm{C} 29-\mathrm{C} 30$ & 1.3900 \\
\hline C6-H6 & 0.9500 & C29-H29 & 0.9500 \\
\hline $\mathrm{C} 7-\mathrm{C} 8$ & 1.3900 & $\mathrm{C} 30-\mathrm{H} 30$ & 0.9500 \\
\hline $\mathrm{C} 7-\mathrm{C} 12$ & 1.3900 & $\mathrm{C} 31-\mathrm{C} 32$ & $1.485(7)$ \\
\hline $\mathrm{C} 8-\mathrm{C} 9$ & 1.3900 & $\mathrm{C} 31-\mathrm{C} 36$ & $1.494(7)$ \\
\hline $\mathrm{C} 8-\mathrm{H} 8$ & 0.9500 & C31-H31 & 1.0000 \\
\hline $\mathrm{C} 9-\mathrm{C} 10$ & 1.3900 & $\mathrm{C} 32-\mathrm{C} 33$ & $1.500(7)$ \\
\hline C9-H9 & 0.9500 & $\mathrm{C} 32-\mathrm{H} 32 \mathrm{~A}$ & 0.9900 \\
\hline $\mathrm{C} 10-\mathrm{C} 11$ & 1.3900 & C $32-\mathrm{H} 32 \mathrm{~B}$ & 0.9900 \\
\hline $\mathrm{C} 10-\mathrm{H} 10$ & 0.9500 & $\mathrm{C} 33-\mathrm{C} 34$ & $1.491(8)$ \\
\hline $\mathrm{C} 11-\mathrm{C} 12$ & 1.3900 & $\mathrm{C} 33-\mathrm{H} 33 \mathrm{~A}$ & 0.9900 \\
\hline $\mathrm{C} 11-\mathrm{H} 11$ & 0.9500 & $\mathrm{C} 33-\mathrm{H} 33 \mathrm{~B}$ & 0.9900 \\
\hline $\mathrm{C} 12-\mathrm{H} 12$ & 0.9500 & C34-C35 & $1.496(8)$ \\
\hline $\mathrm{C} 13-\mathrm{C} 14$ & $1.511(7)$ & $\mathrm{C} 34-\mathrm{H} 34 \mathrm{~A}$ & 0.9900 \\
\hline $\mathrm{C} 13-\mathrm{C} 18$ & $1.523(7)$ & $\mathrm{C} 34-\mathrm{H} 34 \mathrm{~B}$ & 0.9900 \\
\hline $\mathrm{C} 13-\mathrm{H} 13$ & 1.0000 & $\mathrm{C} 35-\mathrm{C} 36$ & $1.496(7)$ \\
\hline $\mathrm{C} 14-\mathrm{C} 15$ & $1.523(7)$ & C $35-\mathrm{H} 35 \mathrm{~A}$ & 0.9900 \\
\hline
\end{tabular}




\begin{tabular}{|c|c|c|c|}
\hline $\mathrm{C} 14-\mathrm{H} 14 \mathrm{~A}$ & 0.9900 & $\mathrm{C} 35-\mathrm{H} 35 \mathrm{C}$ & 0.9900 \\
\hline C14-H14B & 0.9900 & $\mathrm{C} 36-\mathrm{H} 36 \mathrm{C}$ & 0.9900 \\
\hline $\mathrm{C} 15-\mathrm{C} 16$ & $1.526(8)$ & $\mathrm{C} 36-\mathrm{H} 36 \mathrm{~A}$ & 0.9900 \\
\hline $\mathrm{Cu} 1-\mathrm{I} 1-\mathrm{Cu} 1$ & $70.07(4)$ & $\mathrm{C} 13-\mathrm{C} 14-\mathrm{H} 14 \mathrm{~B}$ & 109.3 \\
\hline $\mathrm{Cu} 2-\mathrm{I} 1-\mathrm{Cu} 1^{\mathrm{i}}$ & $68.98(3)$ & $\mathrm{C} 15-\mathrm{C} 14-\mathrm{H} 14 \mathrm{~B}$ & 109.3 \\
\hline $\mathrm{Cu} 2-\mathrm{I} 1-\mathrm{Cu} 1$ & $64.54(3)$ & $\mathrm{H} 14 \mathrm{~A}-\mathrm{C} 14-\mathrm{H} 14 \mathrm{~B}$ & 107.9 \\
\hline $\mathrm{Cu} 2-\mathrm{I} 2-\mathrm{Cu} 1$ & $64.54(3)$ & $\mathrm{C} 14-\mathrm{C} 15-\mathrm{C} 16$ & $111.3(6)$ \\
\hline $\mathrm{Cu} 2-\mathrm{I} 2-\mathrm{Cu} 2^{\mathrm{i}}$ & $68.62(3)$ & $\mathrm{C} 14-\mathrm{C} 15-\mathrm{H} 15 \mathrm{~A}$ & 109.4 \\
\hline $\mathrm{Cu} 1-\mathrm{I} 2-\mathrm{Cu} 2^{\mathrm{i}}$ & $67.18(3)$ & $\mathrm{C} 16-\mathrm{C} 15-\mathrm{H} 15 \mathrm{~A}$ & 109.4 \\
\hline $\mathrm{P} 1-\mathrm{Cu} 1-\mathrm{I} 1^{\mathrm{i}}$ & $112.08(6)$ & $\mathrm{C} 14-\mathrm{C} 15-\mathrm{H} 15 \mathrm{~B}$ & 109.4 \\
\hline $\mathrm{P} 1-\mathrm{Cu} 1-\mathrm{I} 1$ & $111.11(6)$ & $\mathrm{C} 16-\mathrm{C} 15-\mathrm{H} 15 \mathrm{~B}$ & 109.4 \\
\hline $\mathrm{I} 1 \mathrm{i}-\mathrm{Cu} 1-\mathrm{I} 1$ & $103.98(3)$ & $\mathrm{H} 15 \mathrm{~A}-\mathrm{C} 15-\mathrm{H} 15 \mathrm{~B}$ & 108.0 \\
\hline $\mathrm{P} 1-\mathrm{Cu} 1-\mathrm{I} 2$ & $109.39(6)$ & $\mathrm{C} 17-\mathrm{C} 16-\mathrm{C} 15$ & $110.4(6)$ \\
\hline $\mathrm{I} 1^{\mathrm{i}}-\mathrm{Cu} 1-\mathrm{I} 2$ & $107.90(3)$ & $\mathrm{C} 17-\mathrm{C} 16-\mathrm{H} 16 \mathrm{~A}$ & 109.6 \\
\hline $\mathrm{I} 1-\mathrm{Cu} 1-\mathrm{I} 2$ & $112.27(4)$ & $\mathrm{C} 15-\mathrm{C} 16-\mathrm{H} 16 \mathrm{~A}$ & 109.6 \\
\hline $\mathrm{P} 1-\mathrm{Cu} 1-\mathrm{Cu} 2$ & $140.58(6)$ & $\mathrm{C} 17-\mathrm{C} 16-\mathrm{H} 16 \mathrm{~B}$ & 109.6 \\
\hline $\mathrm{I} 1-\mathrm{Cu} 1-\mathrm{Cu} 2$ & $107.34(4)$ & $\mathrm{C} 15-\mathrm{C} 16-\mathrm{H} 16 \mathrm{~B}$ & 109.6 \\
\hline $\mathrm{I} 1-\mathrm{Cu} 1-\mathrm{Cu} 2$ & $57.17(3)$ & $\mathrm{H} 16 \mathrm{~A}-\mathrm{C} 16-\mathrm{H} 16 \mathrm{~B}$ & 108.1 \\
\hline $\mathrm{I} 2-\mathrm{Cu} 1-\mathrm{Cu} 2$ & $56.83(3)$ & $\mathrm{C} 18-\mathrm{C} 17-\mathrm{C} 16$ & $111.1(5)$ \\
\hline $\mathrm{P} 1-\mathrm{Cu} 1-\mathrm{Cu} 2^{\mathrm{i}}$ & $146.32(7)$ & $\mathrm{C} 18-\mathrm{C} 17-\mathrm{H} 17 \mathrm{~A}$ & 109.4 \\
\hline $\mathrm{I} 1^{\mathrm{i}}-\mathrm{Cu} 1-\mathrm{Cu} 2^{\mathrm{i}}$ & $55.19(3)$ & $\mathrm{C} 16-\mathrm{C} 17-\mathrm{H} 17 \mathrm{~A}$ & 109.4 \\
\hline $\mathrm{I} 1-\mathrm{Cu} 1-\mathrm{Cu} 2^{\mathrm{i}}$ & $102.49(4)$ & $\mathrm{C} 18-\mathrm{C} 17-\mathrm{H} 17 \mathrm{~B}$ & 109.4 \\
\hline $\mathrm{I} 2-\mathrm{Cu} 1-\mathrm{Cu} 2^{\mathrm{i}}$ & $57.34(3)$ & $\mathrm{C} 16-\mathrm{C} 17-\mathrm{H} 17 \mathrm{~B}$ & 109.4 \\
\hline $\mathrm{Cu} 2-\mathrm{Cu} 1-\mathrm{Cu}^{2}{ }^{\mathrm{i}}$ & $62.43(4)$ & $\mathrm{H} 17 \mathrm{~A}-\mathrm{C} 17-\mathrm{H} 17 \mathrm{~B}$ & 108.0 \\
\hline $\mathrm{P} 2-\mathrm{Cu} 2-\mathrm{I} 2$ & $118.81(7)$ & $\mathrm{C} 17-\mathrm{C} 18-\mathrm{C} 13$ & $111.4(5)$ \\
\hline $\mathrm{P} 2-\mathrm{Cu} 2-\mathrm{I} 1$ & $106.33(6)$ & $\mathrm{C} 17-\mathrm{C} 18-\mathrm{H} 18 \mathrm{~A}$ & 109.4 \\
\hline $\mathrm{I} 2-\mathrm{Cu} 2-\mathrm{I} 1$ & $115.10(4)$ & $\mathrm{C} 13-\mathrm{C} 18-\mathrm{H} 18 \mathrm{~A}$ & 109.4 \\
\hline $\mathrm{P} 2-\mathrm{Cu} 2-12^{\mathrm{i}}$ & $104.17(6)$ & $\mathrm{C} 17-\mathrm{C} 18-\mathrm{H} 18 \mathrm{~B}$ & 109.4 \\
\hline $\mathrm{I} 2-\mathrm{Cu} 2-\mathrm{I} 2^{\mathrm{i}}$ & $104.49(3)$ & $\mathrm{C} 13-\mathrm{C} 18-\mathrm{H} 18 \mathrm{~B}$ & 109.4 \\
\hline $\mathrm{I} 1-\mathrm{Cu} 2-\mathrm{I} 2^{\mathrm{i}}$ & $106.78(4)$ & $\mathrm{H} 18 \mathrm{~A}-\mathrm{C} 18-\mathrm{H} 18 \mathrm{~B}$ & 108.0 \\
\hline $\mathrm{P} 2-\mathrm{Cu} 2-\mathrm{Cu} 1$ & $147.91(7)$ & $\mathrm{C} 20-\mathrm{C} 19-\mathrm{C} 24$ & 120.0 \\
\hline $\mathrm{I} 2-\mathrm{Cu} 2-\mathrm{Cu} 1$ & $58.64(3)$ & $\mathrm{C} 20-\mathrm{C} 19-\mathrm{P} 2$ & $119.1(3)$ \\
\hline $\mathrm{I} 1-\mathrm{Cu} 2-\mathrm{Cu} 1$ & $58.29(3)$ & $\mathrm{C} 24-\mathrm{C} 19-\mathrm{P} 2$ & $120.7(3)$ \\
\hline $\mathrm{I} 2 \mathrm{i}-\mathrm{Cu} 2-\mathrm{Cu} 1$ & $107.27(4)$ & $\mathrm{C} 19-\mathrm{C} 20-\mathrm{C} 21$ & 120.0 \\
\hline $\mathrm{P} 2-\mathrm{Cu} 2-\mathrm{Cu} 1^{\mathrm{i}}$ & $135.06(7)$ & $\mathrm{C} 19-\mathrm{C} 20-\mathrm{H} 20$ & 120.0 \\
\hline $\mathrm{I} 2-\mathrm{Cu} 2-\mathrm{Cu} 1^{\mathrm{i}}$ & $105.64(4)$ & $\mathrm{C} 21-\mathrm{C} 20-\mathrm{H} 20$ & 120.0 \\
\hline $\mathrm{I} 1-\mathrm{Cu} 2-\mathrm{Cu} 1^{\mathrm{i}}$ & $55.83(3)$ & $\mathrm{C} 20-\mathrm{C} 21-\mathrm{C} 22$ & 120.0 \\
\hline $\mathrm{I} 2^{\mathrm{i}}-\mathrm{Cu} 2-\mathrm{Cu} 1^{\mathrm{i}}$ & $55.48(3)$ & $\mathrm{C} 20-\mathrm{C} 21-\mathrm{H} 21$ & 120.0 \\
\hline $\mathrm{Cu} 1-\mathrm{Cu} 2-\mathrm{Cu} 1^{\mathrm{i}}$ & $63.18(4)$ & $\mathrm{C} 22-\mathrm{C} 21-\mathrm{H} 21$ & 120.0 \\
\hline $\mathrm{P} 2-\mathrm{Cu} 2-\mathrm{Cu} 2^{\mathrm{i}}$ & $148.19(6)$ & $\mathrm{C} 23-\mathrm{C} 22-\mathrm{C} 21$ & 120.0 \\
\hline $\mathrm{I} 2-\mathrm{Cu} 2-\mathrm{Cu} 2^{\mathrm{i}}$ & $57.43(3)$ & $\mathrm{C} 23-\mathrm{C} 22-\mathrm{H} 22$ & 120.0 \\
\hline $\mathrm{I} 1-\mathrm{Cu} 2-\mathrm{Cu} 2^{\mathrm{i}}$ & $102.56(2)$ & $\mathrm{C} 21-\mathrm{C} 22-\mathrm{H} 22$ & 120.0 \\
\hline $\mathrm{I} 2^{\mathrm{i}}-\mathrm{Cu} 2-\mathrm{Cu} 2^{\mathrm{i}}$ & $53.95(3)$ & $\mathrm{C} 22-\mathrm{C} 23-\mathrm{C} 24$ & 120.0 \\
\hline $\mathrm{Cu} 1-\mathrm{Cu} 2-\mathrm{Cu} 2^{\mathrm{i}}$ & $61.45(3)$ & $\mathrm{C} 22-\mathrm{C} 23-\mathrm{H} 23$ & 120.0 \\
\hline $\mathrm{Cu} 1^{\mathrm{i}}-\mathrm{Cu} 2-\mathrm{Cu}_{2}{ }^{\mathrm{i}}$ & $56.12(3)$ & $\mathrm{C} 24-\mathrm{C} 23-\mathrm{H} 23$ & 120.0 \\
\hline $\mathrm{C} 1-\mathrm{P} 1-\mathrm{C} 7$ & $101.0(3)$ & $\mathrm{C} 23-\mathrm{C} 24-\mathrm{C} 19$ & 120.0 \\
\hline $\mathrm{C} 1-\mathrm{P} 1-\mathrm{C} 13$ & $102.6(3)$ & $\mathrm{C} 23-\mathrm{C} 24-\mathrm{H} 24$ & 120.0 \\
\hline
\end{tabular}




\begin{tabular}{|c|c|c|c|}
\hline $\mathrm{C} 7-\mathrm{P} 1-\mathrm{C} 13$ & $104.6(3)$ & $\mathrm{C} 19-\mathrm{C} 24-\mathrm{H} 24$ & 120.0 \\
\hline $\mathrm{C} 1-\mathrm{P} 1-\mathrm{Cu} 1$ & $116.07(18)$ & $\mathrm{C} 26-\mathrm{C} 25-\mathrm{C} 30$ & 120.0 \\
\hline $\mathrm{C} 7-\mathrm{P} 1-\mathrm{Cu} 1$ & $115.1(2)$ & $\mathrm{C} 26-\mathrm{C} 25-\mathrm{P} 2$ & $123.1(3)$ \\
\hline $\mathrm{C} 13-\mathrm{P} 1-\mathrm{Cu} 1$ & $115.6(2)$ & $\mathrm{C} 30-\mathrm{C} 25-\mathrm{P} 2$ & $116.7(3)$ \\
\hline $\mathrm{C} 31-\mathrm{P} 2-\mathrm{C} 19$ & $104.2(3)$ & $\mathrm{C} 27-\mathrm{C} 26-\mathrm{C} 25$ & 120.0 \\
\hline $\mathrm{C} 31-\mathrm{P} 2-\mathrm{C} 25$ & $101.2(4)$ & $\mathrm{C} 27-\mathrm{C} 26-\mathrm{H} 26$ & 120.0 \\
\hline $\mathrm{C} 19-\mathrm{P} 2-\mathrm{C} 25$ & $107.2(3)$ & $\mathrm{C} 25-\mathrm{C} 26-\mathrm{H} 26$ & 120.0 \\
\hline $\mathrm{C} 31-\mathrm{P} 2-\mathrm{Cu} 2$ & $117.8(3)$ & $\mathrm{C} 28-\mathrm{C} 27-\mathrm{C} 26$ & 120.0 \\
\hline $\mathrm{C} 19-\mathrm{P} 2-\mathrm{Cu} 2$ & $109.6(2)$ & $\mathrm{C} 28-\mathrm{C} 27-\mathrm{H} 27$ & 120.0 \\
\hline $\mathrm{C} 25-\mathrm{P} 2-\mathrm{Cu} 2$ & $115.74(18)$ & $\mathrm{C} 26-\mathrm{C} 27-\mathrm{H} 27$ & 120.0 \\
\hline $\mathrm{C} 2-\mathrm{C} 1-\mathrm{C} 6$ & 120.0 & $\mathrm{C} 27-\mathrm{C} 28-\mathrm{C} 29$ & 120.0 \\
\hline $\mathrm{C} 2-\mathrm{C} 1-\mathrm{P} 1$ & $118.7(3)$ & $\mathrm{C} 27-\mathrm{C} 28-\mathrm{H} 28$ & 120.0 \\
\hline $\mathrm{C} 6-\mathrm{C} 1-\mathrm{P} 1$ & $121.3(3)$ & $\mathrm{C} 29-\mathrm{C} 28-\mathrm{H} 28$ & 120.0 \\
\hline $\mathrm{C} 3-\mathrm{C} 2-\mathrm{C} 1$ & 120.0 & $\mathrm{C} 30-\mathrm{C} 29-\mathrm{C} 28$ & 120.0 \\
\hline $\mathrm{C} 3-\mathrm{C} 2-\mathrm{H} 2$ & 120.0 & $\mathrm{C} 30-\mathrm{C} 29-\mathrm{H} 29$ & 120.0 \\
\hline $\mathrm{C} 1-\mathrm{C} 2-\mathrm{H} 2$ & 120.0 & $\mathrm{C} 28-\mathrm{C} 29-\mathrm{H} 29$ & 120.0 \\
\hline $\mathrm{C} 4-\mathrm{C} 3-\mathrm{C} 2$ & 120.0 & $\mathrm{C} 29-\mathrm{C} 30-\mathrm{C} 25$ & 120.0 \\
\hline $\mathrm{C} 4-\mathrm{C} 3-\mathrm{H} 3$ & 120.0 & $\mathrm{C} 29-\mathrm{C} 30-\mathrm{H} 30$ & 120.0 \\
\hline $\mathrm{C} 2-\mathrm{C} 3-\mathrm{H} 3$ & 120.0 & $\mathrm{C} 25-\mathrm{C} 30-\mathrm{H} 30$ & 120.0 \\
\hline $\mathrm{C} 5-\mathrm{C} 4-\mathrm{C} 3$ & 120.0 & $\mathrm{C} 32-\mathrm{C} 31-\mathrm{C} 36$ & $115.4(5)$ \\
\hline $\mathrm{C} 5-\mathrm{C} 4-\mathrm{H} 4$ & 120.0 & $\mathrm{C} 32-\mathrm{C} 31-\mathrm{P} 2$ & $118.2(5)$ \\
\hline $\mathrm{C} 3-\mathrm{C} 4-\mathrm{H} 4$ & 120.0 & $\mathrm{C} 36-\mathrm{C} 31-\mathrm{P} 2$ & $113.9(5)$ \\
\hline $\mathrm{C} 4-\mathrm{C} 5-\mathrm{C} 6$ & 120.0 & $\mathrm{C} 32-\mathrm{C} 31-\mathrm{H} 31$ & 101.9 \\
\hline $\mathrm{C} 4-\mathrm{C} 5-\mathrm{H} 5$ & 120.0 & $\mathrm{C} 36-\mathrm{C} 31-\mathrm{H} 31$ & 101.9 \\
\hline $\mathrm{C} 6-\mathrm{C} 5-\mathrm{H} 5$ & 120.0 & $\mathrm{P} 2-\mathrm{C} 31-\mathrm{H} 31$ & 101.9 \\
\hline $\mathrm{C} 5-\mathrm{C} 6-\mathrm{C} 1$ & 120.0 & $\mathrm{C} 31-\mathrm{C} 32-\mathrm{C} 33$ & $115.5(6)$ \\
\hline $\mathrm{C} 5-\mathrm{C} 6-\mathrm{H} 6$ & 120.0 & $\mathrm{C} 31-\mathrm{C} 32-\mathrm{H} 32 \mathrm{~A}$ & 108.4 \\
\hline $\mathrm{C} 1-\mathrm{C} 6-\mathrm{H} 6$ & 120.0 & $\mathrm{C} 33-\mathrm{C} 32-\mathrm{H} 32 \mathrm{~A}$ & 108.4 \\
\hline $\mathrm{C} 8-\mathrm{C} 7-\mathrm{C} 12$ & 120.0 & $\mathrm{C} 31-\mathrm{C} 32-\mathrm{H} 32 \mathrm{~B}$ & 108.4 \\
\hline $\mathrm{C} 8-\mathrm{C} 7-\mathrm{P} 1$ & $115.7(3)$ & $\mathrm{C} 33-\mathrm{C} 32-\mathrm{H} 32 \mathrm{~B}$ & 108.4 \\
\hline $\mathrm{C} 12-\mathrm{C} 7-\mathrm{P} 1$ & $124.2(3)$ & $\mathrm{H} 32 \mathrm{~A}-\mathrm{C} 32-\mathrm{H} 32 \mathrm{~B}$ & 107.5 \\
\hline $\mathrm{C} 9-\mathrm{C} 8-\mathrm{C} 7$ & 120.0 & $\mathrm{C} 34-\mathrm{C} 33-\mathrm{C} 32$ & $115.1(6)$ \\
\hline $\mathrm{C} 9-\mathrm{C} 8-\mathrm{H} 8$ & 120.0 & C34-C33-H33A & 108.5 \\
\hline $\mathrm{C} 7-\mathrm{C} 8-\mathrm{H} 8$ & 120.0 & $\mathrm{C} 32-\mathrm{C} 33-\mathrm{H} 33 \mathrm{~A}$ & 108.5 \\
\hline $\mathrm{C} 10-\mathrm{C} 9-\mathrm{C} 8$ & 120.0 & C $34-\mathrm{C} 33-\mathrm{H} 33 \mathrm{~B}$ & 108.5 \\
\hline $\mathrm{C} 10-\mathrm{C} 9-\mathrm{H} 9$ & 120.0 & $\mathrm{C} 32-\mathrm{C} 33-\mathrm{H} 33 \mathrm{~B}$ & 108.5 \\
\hline $\mathrm{C} 8-\mathrm{C} 9-\mathrm{H} 9$ & 120.0 & $\mathrm{H} 33 \mathrm{~A}-\mathrm{C} 33-\mathrm{H} 33 \mathrm{~B}$ & 107.5 \\
\hline $\mathrm{C} 9-\mathrm{C} 10-\mathrm{C} 11$ & 120.0 & $\mathrm{C} 33-\mathrm{C} 34-\mathrm{C} 35$ & $114.8(6)$ \\
\hline $\mathrm{C} 9-\mathrm{C} 10-\mathrm{H} 10$ & 120.0 & $\mathrm{C} 33-\mathrm{C} 34-\mathrm{H} 34 \mathrm{~A}$ & 108.6 \\
\hline $\mathrm{C} 11-\mathrm{C} 10-\mathrm{H} 10$ & 120.0 & $\mathrm{C} 35-\mathrm{C} 34-\mathrm{H} 34 \mathrm{~A}$ & 108.6 \\
\hline $\mathrm{C} 10-\mathrm{C} 11-\mathrm{C} 12$ & 120.0 & $\mathrm{C} 33-\mathrm{C} 34-\mathrm{H} 34 \mathrm{~B}$ & 108.6 \\
\hline $\mathrm{C} 10-\mathrm{C} 11-\mathrm{H} 11$ & 120.0 & $\mathrm{C} 35-\mathrm{C} 34-\mathrm{H} 34 \mathrm{~B}$ & 108.6 \\
\hline $\mathrm{C} 12-\mathrm{C} 11-\mathrm{H} 11$ & 120.0 & $\mathrm{H} 34 \mathrm{~A}-\mathrm{C} 34-\mathrm{H} 34 \mathrm{~B}$ & 107.6 \\
\hline $\mathrm{C} 11-\mathrm{C} 12-\mathrm{C} 7$ & 120.0 & $\mathrm{C} 36-\mathrm{C} 35-\mathrm{C} 34$ & $114.3(6)$ \\
\hline $\mathrm{C} 11-\mathrm{C} 12-\mathrm{H} 12$ & 120.0 & $\mathrm{C} 36-\mathrm{C} 35-\mathrm{H} 35 \mathrm{~A}$ & 108.7 \\
\hline $\mathrm{C} 7-\mathrm{C} 12-\mathrm{H} 12$ & 120.0 & $\mathrm{C} 34-\mathrm{C} 35-\mathrm{H} 35 \mathrm{~A}$ & 108.7 \\
\hline $\mathrm{C} 14-\mathrm{C} 13-\mathrm{C} 18$ & $111.7(5)$ & $\mathrm{C} 36-\mathrm{C} 35-\mathrm{H} 35 \mathrm{C}$ & 108.7 \\
\hline $\mathrm{C} 14-\mathrm{C} 13-\mathrm{P} 1$ & $112.6(4)$ & $\mathrm{C} 34-\mathrm{C} 35-\mathrm{H} 35 \mathrm{C}$ & 108.7 \\
\hline
\end{tabular}


C18-C13-P1

C14-C13-H13

$\mathrm{C} 18-\mathrm{C} 13-\mathrm{H} 13$

$\mathrm{P} 1-\mathrm{C} 13-\mathrm{H} 13$

$\mathrm{C} 13-\mathrm{C} 14-\mathrm{C} 15$

C13-C14-H14A

C15-C14-H14A

$\mathrm{Cu} 2-\mathrm{I} 1-\mathrm{Cu} 1-\mathrm{P} 1$

$\mathrm{Cu} 1$ i- I1-Cu1-P1

$\mathrm{Cu} 2-\mathrm{I} 1-\mathrm{Cu} 1-\mathrm{I}^{\mathrm{i}}$

$\mathrm{Cu} 1-\mathrm{I} 1-\mathrm{Cu} 1-\mathrm{I}^{\mathrm{i}}$

$\mathrm{Cu} 2-\mathrm{I} 1-\mathrm{Cu} 1-\mathrm{I} 2$

$\mathrm{Cu} 1$ i- I1- Cu1- 2

$\mathrm{Cu} 1$ i $-\mathrm{I} 1-\mathrm{Cu} 1-\mathrm{Cu} 2$

$\mathrm{Cu} 2-\mathrm{I} 1-\mathrm{Cu} 1-\mathrm{Cu} 2$

$\mathrm{Cu} 1-\mathrm{I} 1-\mathrm{Cu} 1-\mathrm{Cu}^{2}{ }^{\mathrm{i}}$

$\mathrm{Cu} 2-\mathrm{I} 2-\mathrm{Cu} 1-\mathrm{P} 1$

$\mathrm{Cu} 2-\mathrm{I} 2-\mathrm{Cu} 1-\mathrm{P} 1$

$\mathrm{Cu} 2-\mathrm{I} 2-\mathrm{Cu} 1-\mathrm{I}^{\mathrm{i}}$

$\mathrm{Cu} 2^{\mathrm{i}}-\mathrm{I} 2-\mathrm{Cu} 1-\mathrm{I}^{\mathrm{i}}$

$\mathrm{Cu} 2-\mathrm{I} 2-\mathrm{Cu} 1-\mathrm{I} 1$

$\mathrm{Cu} 2 \mathrm{i}-\mathrm{I} 2-\mathrm{Cu} 1-\mathrm{I} 1$

$\mathrm{Cu} 2 \mathrm{i}-\mathrm{I} 2-\mathrm{Cu} 1-\mathrm{Cu} 2$

$\mathrm{Cu} 2-\mathrm{I} 2-\mathrm{Cu} 1-\mathrm{Cu} 2^{\mathrm{i}}$

$\mathrm{Cu} 1-\mathrm{I} 2-\mathrm{Cu} 2-\mathrm{P} 2$

$\mathrm{Cu} 2-\mathrm{I} 2-\mathrm{Cu} 2-\mathrm{P} 2$

$\mathrm{Cu} 1-\mathrm{I} 2-\mathrm{Cu} 2-\mathrm{I} 1$

$\mathrm{Cu} 2 \mathrm{i}-\mathrm{I} 2-\mathrm{Cu} 2-\mathrm{I} 1$

$\mathrm{Cu} 1-\mathrm{I} 2-\mathrm{Cu} 2-\mathrm{I} 2^{\mathrm{i}}$

$\mathrm{Cu} 2^{\mathrm{i}}-\mathrm{I} 2-\mathrm{Cu} 2-\mathrm{I}^{\mathrm{i}}$

$\mathrm{Cu} 2 \mathrm{i}-\mathrm{I} 2-\mathrm{Cu} 2-\mathrm{Cu} 1$

$\mathrm{Cu} 1-\mathrm{I} 2-\mathrm{Cu} 2-\mathrm{Cu}^{\mathrm{i}}$

$\mathrm{Cu} 2-\mathrm{i} 2-\mathrm{Cu} 2-\mathrm{Cu}^{\mathrm{i}}{ }^{\mathrm{i}}$

$\mathrm{Cu} 1-\mathrm{I} 2-\mathrm{Cu} 2-\mathrm{Cu} 2^{\mathrm{i}}$

$\mathrm{Cu} 1-\mathrm{I} 1-\mathrm{Cu} 2-\mathrm{P} 2$

$\mathrm{Cu} 1-\mathrm{I} 1-\mathrm{Cu} 2-\mathrm{P} 2$

$\mathrm{Cu} 1-\mathrm{I} 1-\mathrm{Cu} 2-\mathrm{I} 2$

$\mathrm{Cu} 1-\mathrm{I} 1-\mathrm{Cu} 2-\mathrm{I} 2$

$\mathrm{Cu} 1-\mathrm{I} 1-\mathrm{Cu} 2-\mathrm{I}^{\mathrm{i}}$

$\mathrm{Cu} 1-\mathrm{I} 1-\mathrm{Cu} 2-\mathrm{I}^{\mathrm{i}}$

$\mathrm{Cu} 1-\mathrm{I} 1-\mathrm{Cu} 2-\mathrm{Cu} 1$

$\mathrm{Cu} 1-\mathrm{I} 1-\mathrm{Cu} 2-\mathrm{Cu}^{\mathrm{i}}$

$\mathrm{Cu} 1-\mathrm{I} 1-\mathrm{Cu} 2-\mathrm{Cu}^{2}{ }^{\mathrm{i}}$

$\mathrm{Cu} 1-\mathrm{I} 1-\mathrm{Cu} 2-\mathrm{Cu} 2^{\mathrm{i}}$

$\mathrm{P} 1-\mathrm{Cu} 1-\mathrm{Cu} 2-\mathrm{P} 2$

$\mathrm{I} 1-\mathrm{Cu} 1-\mathrm{Cu} 2-\mathrm{P} 2$

$\mathrm{I} 1-\mathrm{Cu} 1-\mathrm{Cu} 2-\mathrm{P} 2$
$111.2(4)$

107.0

107.0

107.0

111.8 (6)

109.3

109.3

$137.42(7)$

-147.05 (6)

-101.82 (4)

-26.29 (4)

$14.56(3)$

90.08 (3)

75.53 (3)

-45.01 (4)

30.52 (4)

$-138.44(7)$

$145.31(7)$

99.38 (4)

23.13 (3)

-14.61 (3)

-90.86 (4)

-76.25 (4)

76.25 (4)

$142.86(8)$

$-143.09(7)$

15.13 (3)

89.18 (3)

-101.63 (4)

-27.58 (4)

74.05 (3)

-44.01 (4)

30.04 (4)

-74.05 (3)

$133.84(7)$

$-148.96(7)$

-92.39 (4)

-15.19 (3)

23.07 (3)

100.27 (4)

$-77.20(4)$

77.20 (4)

-32.70 (4)

44.50 (4)

-14.99 (18)

164.42 (12)

$68.70(13)$
$\mathrm{H} 35 \mathrm{~A}-\mathrm{C} 35-\mathrm{H} 35 \mathrm{C}$

$\mathrm{C} 31-\mathrm{C} 36-\mathrm{C} 35$

$\mathrm{C} 31-\mathrm{C} 36-\mathrm{H} 36 \mathrm{C}$

$\mathrm{C} 35-\mathrm{C} 36-\mathrm{H} 36 \mathrm{C}$

C31-C36- H36A

$\mathrm{C} 35-\mathrm{C} 36-\mathrm{H} 36 \mathrm{~A}$

$\mathrm{H} 36 \mathrm{C}-\mathrm{C} 36-\mathrm{H} 36 \mathrm{~A}$

$\mathrm{I} 2-\mathrm{Cu} 2-\mathrm{P} 2-\mathrm{C} 25$

$\mathrm{I} 1-\mathrm{Cu} 2-\mathrm{P} 2-\mathrm{C} 25$

$\mathrm{I} 2 \mathrm{i}-\mathrm{Cu} 2-\mathrm{P} 2-\mathrm{C} 25$

$\mathrm{Cu} 1-\mathrm{Cu} 2-\mathrm{P} 2-\mathrm{C} 25$

$\mathrm{Cu} 1-\mathrm{Cu} 2-\mathrm{P} 2-\mathrm{C} 25$

$\mathrm{Cu} 2 \mathrm{i}-\mathrm{Cu} 2-\mathrm{P} 2-\mathrm{C} 25$

$\mathrm{C} 7-\mathrm{P} 1-\mathrm{C} 1-\mathrm{C} 2$

$\mathrm{C} 13-\mathrm{P} 1-\mathrm{C} 1-\mathrm{C} 2$

$\mathrm{Cu} 1-\mathrm{P} 1-\mathrm{C} 1-\mathrm{C} 2$

$\mathrm{C} 7-\mathrm{P} 1-\mathrm{C} 1-\mathrm{C} 6$

$\mathrm{C} 13-\mathrm{P} 1-\mathrm{C} 1-\mathrm{C} 6$

$\mathrm{Cu} 1-\mathrm{P} 1-\mathrm{C} 1-\mathrm{C} 6$

$\mathrm{C} 6-\mathrm{C} 1-\mathrm{C} 2-\mathrm{C} 3$

$\mathrm{P} 1-\mathrm{C} 1-\mathrm{C} 2-\mathrm{C} 3$

$\mathrm{C} 1-\mathrm{C} 2-\mathrm{C} 3-\mathrm{C} 4$

$\mathrm{C} 2-\mathrm{C} 3-\mathrm{C} 4-\mathrm{C} 5$

$\mathrm{C} 3-\mathrm{C} 4-\mathrm{C} 5-\mathrm{C} 6$

$\mathrm{C} 4-\mathrm{C} 5-\mathrm{C} 6-\mathrm{C} 1$

$\mathrm{C} 2-\mathrm{C} 1-\mathrm{C} 6-\mathrm{C} 5$

$\mathrm{P} 1-\mathrm{C} 1-\mathrm{C} 6-\mathrm{C} 5$

$\mathrm{C} 1-\mathrm{P} 1-\mathrm{C} 7-\mathrm{C} 8$

$\mathrm{C} 13-\mathrm{P} 1-\mathrm{C} 7-\mathrm{C} 8$

$\mathrm{Cu} 1-\mathrm{P} 1-\mathrm{C} 7-\mathrm{C} 8$

C1-P1-C7-C12

$\mathrm{C} 13-\mathrm{P} 1-\mathrm{C} 7-\mathrm{C} 12$

$\mathrm{Cu} 1-\mathrm{P} 1-\mathrm{C} 7-\mathrm{C} 12$

$\mathrm{C} 12-\mathrm{C} 7-\mathrm{C} 8-\mathrm{C} 9$

$\mathrm{P} 1-\mathrm{C} 7-\mathrm{C} 8-\mathrm{C} 9$

$\mathrm{C} 7-\mathrm{C} 8-\mathrm{C} 9-\mathrm{C} 10$

$\mathrm{C} 8-\mathrm{C} 9-\mathrm{C} 10-\mathrm{C} 11$

$\mathrm{C} 9-\mathrm{C} 10-\mathrm{C} 11-\mathrm{C} 12$

$\mathrm{C} 10-\mathrm{C} 11-\mathrm{C} 12-\mathrm{C} 7$

$\mathrm{C} 8-\mathrm{C} 7-\mathrm{C} 12-\mathrm{C} 11$

$\mathrm{P} 1-\mathrm{C} 7-\mathrm{C} 12-\mathrm{C} 11$

$\mathrm{C} 1-\mathrm{P} 1-\mathrm{C} 13-\mathrm{C} 14$

$\mathrm{C} 7-\mathrm{P} 1-\mathrm{C} 13-\mathrm{C} 14$

$\mathrm{Cu} 1-\mathrm{P} 1-\mathrm{C} 13-\mathrm{C} 14$

$\mathrm{C} 1-\mathrm{P} 1-\mathrm{C} 13-\mathrm{C} 18$

C7-P1-C13-C18

$\mathrm{Cu} 1-\mathrm{P} 1-\mathrm{C} 13-\mathrm{C} 18$
107.6

115.9 (5)

108.3

108.3

108.3

108.3

107.4

$62.0(2)$

-166.3 (2)

$-53.7(2)$

138.1 (2)

-108.6 (2)

-11.8 (3)

140.6 (3)

$-111.6(4)$

15.5 (4)

-40.3 (4)

67.5 (4)

-165.4 (2)

0.0

179.1 (4)

0.0

0.0

0.0

0.0

0.0

-179.1 (4)

-61.8 (4)

-168.0 (3)

64.0 (3)

115.8 (4)

9.6 (4)

-118.4 (3)

0.0

177.7 (4)

0.0

0.0

0.0

0.0

0.0

-177.5 (5)

46.0 (6)

$151.1(5)$

$-81.3(6)$

172.3 (5)

$-82.7(5)$

$45.0(5)$ 


\begin{tabular}{|c|c|c|c|}
\hline $\mathrm{I} 2-\mathrm{Cu} 1-\mathrm{Cu} 2-\mathrm{P} 2$ & $-95.17(13)$ & $\mathrm{C} 18-\mathrm{C} 13-\mathrm{C} 14-\mathrm{C} 15$ & $53.7(9)$ \\
\hline $\mathrm{Cu} 2-\mathrm{Cu} 1-\mathrm{Cu} 2-\mathrm{P} 2$ & $-162.46(14)$ & $\mathrm{P} 1-\mathrm{C} 13-\mathrm{C} 14-\mathrm{C} 15$ & $179.7(6)$ \\
\hline $\mathrm{P} 1-\mathrm{Cu} 1-\mathrm{Cu} 2-\mathrm{I} 2$ & $80.18(10)$ & $\mathrm{C} 13-\mathrm{C} 14-\mathrm{C} 15-\mathrm{C} 16$ & $-54.8(11)$ \\
\hline 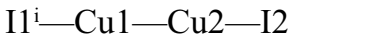 & $-100.41(4)$ & $\mathrm{C} 14-\mathrm{C} 15-\mathrm{C} 16-\mathrm{C} 17$ & $56.0(11)$ \\
\hline $\mathrm{I} 1-\mathrm{Cu} 1-\mathrm{Cu} 2-\mathrm{I} 2$ & $163.87(4)$ & $\mathrm{C} 15-\mathrm{C} 16-\mathrm{C} 17-\mathrm{C} 18$ & $-56.7(10)$ \\
\hline $\mathrm{Cu} 2-\mathrm{Cu} 1-\mathrm{Cu} 2-\mathrm{I} 2$ & $-67.29(3)$ & $\mathrm{C} 16-\mathrm{C} 17-\mathrm{C} 18-\mathrm{C} 13$ & $55.9(9)$ \\
\hline $\mathrm{P} 1-\mathrm{Cu} 1-\mathrm{Cu} 2-\mathrm{I} 1$ & $-83.69(10)$ & $\mathrm{C} 14-\mathrm{C} 13-\mathrm{C} 18-\mathrm{C} 17$ & $-54.3(8)$ \\
\hline $\mathrm{I} 11^{\mathrm{i}}-\mathrm{Cu} 1-\mathrm{Cu} 2-\mathrm{I} 1$ & $95.73(4)$ & $\mathrm{P} 1-\mathrm{C} 13-\mathrm{C} 18-\mathrm{C} 17$ & $179.0(5)$ \\
\hline $\mathrm{I} 2-\mathrm{Cu} 1-\mathrm{Cu} 2-\mathrm{I} 1$ & $-163.87(4)$ & $\mathrm{C} 31-\mathrm{P} 2-\mathrm{C} 19-\mathrm{C} 20$ & $-44.7(4)$ \\
\hline $\mathrm{Cu} 2-\mathrm{Cu} 1-\mathrm{Cu} 2-\mathrm{I} 1$ & $128.84(4)$ & $\mathrm{C} 25-\mathrm{P} 2-\mathrm{C} 19-\mathrm{C} 20$ & $-151.5(3)$ \\
\hline $\mathrm{P} 1-\mathrm{Cu} 1-\mathrm{Cu} 2-\mathrm{I} 2^{\mathrm{i}}$ & $176.92(9)$ & $\mathrm{Cu} 2-\mathrm{P} 2-\mathrm{C} 19-\mathrm{C} 20$ & $82.2(3)$ \\
\hline $\mathrm{I} 1^{\mathrm{i}}-\mathrm{Cu} 1-\mathrm{Cu} 2-\mathrm{I} 2^{\mathrm{i}}$ & $-3.67(5)$ & $\mathrm{C} 31-\mathrm{P} 2-\mathrm{C} 19-\mathrm{C} 24$ & $141.2(4)$ \\
\hline $\mathrm{I} 1-\mathrm{Cu} 1-\mathrm{Cu} 2-\mathrm{I}^{\mathrm{i}}$ & $-99.39(4)$ & $\mathrm{C} 25-\mathrm{P} 2-\mathrm{C} 19-\mathrm{C} 24$ & $34.5(4)$ \\
\hline $\mathrm{I} 2-\mathrm{Cu} 1-\mathrm{Cu} 2-\mathrm{I} 2^{\mathrm{i}}$ & $96.74(4)$ & $\mathrm{Cu} 2-\mathrm{P} 2-\mathrm{C} 19-\mathrm{C} 24$ & $-91.8(3)$ \\
\hline $\mathrm{Cu} 2^{\mathrm{i}}-\mathrm{Cu} 1-\mathrm{Cu} 2-\mathrm{I}^{\mathrm{i}}$ & $29.45(3)$ & $\mathrm{C} 24-\mathrm{C} 19-\mathrm{C} 20-\mathrm{C} 21$ & 0.0 \\
\hline $\mathrm{P} 1-\mathrm{Cu} 1-\mathrm{Cu} 2-\mathrm{Cu} 1^{\mathrm{i}}$ & $-148.38(11)$ & $\mathrm{P} 2-\mathrm{C} 19-\mathrm{C} 20-\mathrm{C} 21$ & $-174.1(4)$ \\
\hline $\mathrm{I} 1^{\mathrm{i}}-\mathrm{Cu} 1-\mathrm{Cu} 2-\mathrm{Cu} 1^{\mathrm{i}}$ & $31.03(4)$ & $\mathrm{C} 19-\mathrm{C} 20-\mathrm{C} 21-\mathrm{C} 22$ & 0.0 \\
\hline $\mathrm{I} 1-\mathrm{Cu} 1-\mathrm{Cu} 2-\mathrm{Cu} 1^{\mathrm{i}}$ & $-64.69(3)$ & $\mathrm{C} 20-\mathrm{C} 21-\mathrm{C} 22-\mathrm{C} 23$ & 0.0 \\
\hline $\mathrm{I} 2-\mathrm{Cu} 1-\mathrm{Cu} 2-\mathrm{Cu} 1^{\mathrm{i}}$ & $131.44(4)$ & $\mathrm{C} 21-\mathrm{C} 22-\mathrm{C} 23-\mathrm{C} 24$ & 0.0 \\
\hline $\mathrm{Cu} 2^{\mathrm{i}}-\mathrm{Cu} 1-\mathrm{Cu} 2-\mathrm{Cu} 1^{\mathrm{i}}$ & $64.15(4)$ & $\mathrm{C} 22-\mathrm{C} 23-\mathrm{C} 24-\mathrm{C} 19$ & 0.0 \\
\hline $\mathrm{P} 1-\mathrm{Cu} 1-\mathrm{Cu} 2-\mathrm{Cu} 2^{\mathrm{i}}$ & $147.47(11)$ & $\mathrm{C} 20-\mathrm{C} 19-\mathrm{C} 24-\mathrm{C} 23$ & 0.0 \\
\hline $\mathrm{I} 1^{\mathrm{i}}-\mathrm{Cu} 1-\mathrm{Cu} 2-\mathrm{Cu}^{2}$ & $-33.12(4)$ & $\mathrm{P} 2-\mathrm{C} 19-\mathrm{C} 24-\mathrm{C} 23$ & $174.0(4)$ \\
\hline $\mathrm{I} 1-\mathrm{Cu} 1-\mathrm{Cu} 2-\mathrm{Cu} 2^{\mathrm{i}}$ & $-128.84(4)$ & $\mathrm{C} 31-\mathrm{P} 2-\mathrm{C} 25-\mathrm{C} 26$ & $-71.7(4)$ \\
\hline $\mathrm{I} 2-\mathrm{Cu} 1-\mathrm{Cu} 2-\mathrm{Cu} 2^{\mathrm{i}}$ & $67.29(3)$ & $\mathrm{C} 19-\mathrm{P} 2-\mathrm{C} 25-\mathrm{C} 26$ & $37.2(4)$ \\
\hline $\mathrm{I} 1{ }^{\mathrm{i}}-\mathrm{Cu} 1-\mathrm{P} 1-\mathrm{C} 1$ & $166.9(2)$ & $\mathrm{Cu} 2-\mathrm{P} 2-\mathrm{C} 25-\mathrm{C} 26$ & $159.8(3)$ \\
\hline $\mathrm{I} 1-\mathrm{Cu} 1-\mathrm{P} 1-\mathrm{C} 1$ & $-77.2(2)$ & $\mathrm{C} 31-\mathrm{P} 2-\mathrm{C} 25-\mathrm{C} 30$ & $103.8(4)$ \\
\hline $\mathrm{I} 2-\mathrm{Cu} 1-\mathrm{P} 1-\mathrm{C} 1$ & $47.3(2)$ & $\mathrm{C} 19-\mathrm{P} 2-\mathrm{C} 25-\mathrm{C} 30$ & $-147.3(3)$ \\
\hline $\mathrm{Cu} 2-\mathrm{Cu} 1-\mathrm{P} 1-\mathrm{C} 1$ & $-13.7(2)$ & $\mathrm{Cu} 2-\mathrm{P} 2-\mathrm{C} 25-\mathrm{C} 30$ & $-24.7(4)$ \\
\hline $\mathrm{Cu} 2^{\mathrm{i}}-\mathrm{Cu} 1-\mathrm{P} 1-\mathrm{C} 1$ & $107.0(2)$ & $\mathrm{C} 30-\mathrm{C} 25-\mathrm{C} 26-\mathrm{C} 27$ & 0.0 \\
\hline $\mathrm{I} 1-\mathrm{Cu} 1-\mathrm{P} 1-\mathrm{C} 7$ & $49.3(2)$ & $\mathrm{P} 2-\mathrm{C} 25-\mathrm{C} 26-\mathrm{C} 27$ & $175.4(5)$ \\
\hline $\mathrm{I} 1-\mathrm{Cu} 1-\mathrm{P} 1-\mathrm{C} 7$ & $165.2(2)$ & $\mathrm{C} 25-\mathrm{C} 26-\mathrm{C} 27-\mathrm{C} 28$ & 0.0 \\
\hline $\mathrm{I} 2-\mathrm{Cu} 1-\mathrm{P} 1-\mathrm{C} 7$ & $-70.3(2)$ & $\mathrm{C} 26-\mathrm{C} 27-\mathrm{C} 28-\mathrm{C} 29$ & 0.0 \\
\hline $\mathrm{Cu} 2-\mathrm{Cu} 1-\mathrm{P} 1-\mathrm{C} 7$ & $-131.3(2)$ & $\mathrm{C} 27-\mathrm{C} 28-\mathrm{C} 29-\mathrm{C} 30$ & 0.0 \\
\hline $\mathrm{Cu} 2^{\mathrm{i}}-\mathrm{Cu} 1-\mathrm{P} 1-\mathrm{C} 7$ & $-10.6(3)$ & $\mathrm{C} 28-\mathrm{C} 29-\mathrm{C} 30-\mathrm{C} 25$ & 0.0 \\
\hline $\mathrm{I} 1{ }^{\mathrm{i}}-\mathrm{Cu} 1-\mathrm{P} 1-\mathrm{C} 13$ & $-72.9(2)$ & $\mathrm{C} 26-\mathrm{C} 25-\mathrm{C} 30-\mathrm{C} 29$ & 0.0 \\
\hline $\mathrm{I} 1-\mathrm{Cu} 1-\mathrm{P} 1-\mathrm{C} 13$ & $43.0(2)$ & $\mathrm{P} 2-\mathrm{C} 25-\mathrm{C} 30-\mathrm{C} 29$ & $-175.6(4)$ \\
\hline $\mathrm{I} 2-\mathrm{Cu} 1-\mathrm{P} 1-\mathrm{C} 13$ & $167.5(2)$ & $\mathrm{C} 19-\mathrm{P} 2-\mathrm{C} 31-\mathrm{C} 32$ & $-41.9(8)$ \\
\hline $\mathrm{Cu} 2-\mathrm{Cu} 1-\mathrm{P} 1-\mathrm{C} 13$ & $106.5(2)$ & $\mathrm{C} 25-\mathrm{P} 2-\mathrm{C} 31-\mathrm{C} 32$ & $69.2(7)$ \\
\hline $\mathrm{Cu} 2-\mathrm{Cu} 1-\mathrm{P} 1-\mathrm{C} 13$ & $-132.7(2)$ & $\mathrm{Cu} 2-\mathrm{P} 2-\mathrm{C} 31-\mathrm{C} 32$ & $-163.6(6)$ \\
\hline $\mathrm{I} 2-\mathrm{Cu} 2-\mathrm{P} 2-\mathrm{C} 31$ & $-57.8(3)$ & $\mathrm{C} 19-\mathrm{P} 2-\mathrm{C} 31-\mathrm{C} 36$ & $177.8(6)$ \\
\hline $\mathrm{I} 1-\mathrm{Cu} 2-\mathrm{P} 2-\mathrm{C} 31$ & $73.9(3)$ & $\mathrm{C} 25-\mathrm{P} 2-\mathrm{C} 31-\mathrm{C} 36$ & $-71.1(6)$ \\
\hline $\mathrm{I} 2-\mathrm{Cu} 2-\mathrm{P} 2-\mathrm{C} 31$ & $-173.5(3)$ & $\mathrm{Cu} 2-\mathrm{P} 2-\mathrm{C} 31-\mathrm{C} 36$ & $56.1(7)$ \\
\hline $\mathrm{Cu} 1-\mathrm{Cu} 2-\mathrm{P} 2-\mathrm{C} 31$ & $18.2(4)$ & $\mathrm{C} 36-\mathrm{C} 31-\mathrm{C} 32-\mathrm{C} 33$ & $-40.0(11)$ \\
\hline $\mathrm{Cu} 1-\mathrm{Cu} 2-\mathrm{P} 2-\mathrm{C} 31$ & $131.5(3)$ & $\mathrm{P} 2-\mathrm{C} 31-\mathrm{C} 32-\mathrm{C} 33$ & $-179.8(7)$ \\
\hline $\mathrm{Cu} 22^{\mathrm{i}}-\mathrm{Cu} 2-\mathrm{P} 2-\mathrm{C} 31$ & $-131.6(3)$ & $\mathrm{C} 31-\mathrm{C} 32-\mathrm{C} 33-\mathrm{C} 34$ & $42.1(11)$ \\
\hline $\mathrm{I} 2-\mathrm{Cu} 2-\mathrm{P} 2-\mathrm{C} 19$ & $-176.71(19)$ & $\mathrm{C} 32-\mathrm{C} 33-\mathrm{C} 34-\mathrm{C} 35$ & $-44.2(12)$ \\
\hline $\mathrm{I} 1-\mathrm{Cu} 2-\mathrm{P} 2-\mathrm{C} 19$ & $-45.0(2)$ & $\mathrm{C} 33-\mathrm{C} 34-\mathrm{C} 35-\mathrm{C} 36$ & $44.2(12)$ \\
\hline $\mathrm{I} 2-\mathrm{Cu} 2-\mathrm{P} 2-\mathrm{C} 19$ & $67.6(2)$ & $\mathrm{C} 32-\mathrm{C} 31-\mathrm{C} 36-\mathrm{C} 35$ & $40.6(11)$ \\
\hline
\end{tabular}




$\begin{array}{llll}\mathrm{Cu} 1-\mathrm{Cu} 2-\mathrm{P} 2-\mathrm{C} 19 & -100.7(2) & \mathrm{P} 2-\mathrm{C} 31-\mathrm{C} 36-\mathrm{C} 35 & -177.9(7) \\ \mathrm{Cu} 1-\mathrm{C} u 2-\mathrm{P} 2-\mathrm{C} 19 & 12.7(2) & \mathrm{C} 34-\mathrm{C} 35-\mathrm{C} 36-\mathrm{C} 31 & -42.5(12) \\ \mathrm{Cu} 2-\mathrm{Cu} 2-\mathrm{P} 2-\mathrm{C} 19 & 109.5(2) & & \end{array}$

Symmetry code: (i) $-x+1, y,-z+3 / 2$. 